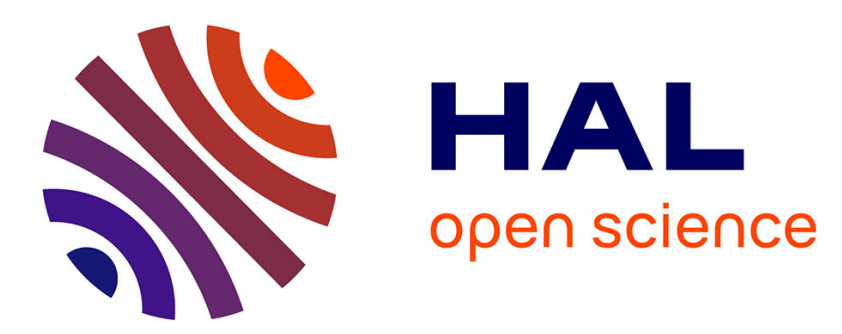

\title{
Nosé-Andersen dynamics of partially rigid molecules: Coupling all degrees of freedom to heat and pressure baths
}

\author{
G. Kneller, T. Mülders
}

\section{To cite this version:}

G. Kneller, T. Mülders. Nosé-Andersen dynamics of partially rigid molecules: Coupling all degrees of freedom to heat and pressure baths. Physical Review E , 1997, 54 (6), pp.6825-6837. 10.1103/PhysRevE.54.6825 . hal-02155694

\section{HAL Id: hal-02155694 \\ https://hal.science/hal-02155694}

Submitted on 13 Jun 2019

HAL is a multi-disciplinary open access archive for the deposit and dissemination of scientific research documents, whether they are published or not. The documents may come from teaching and research institutions in France or abroad, or from public or private research centers.
L'archive ouverte pluridisciplinaire HAL, est destinée au dépôt et à la diffusion de documents scientifiques de niveau recherche, publiés ou non, émanant des établissements d'enseignement et de recherche français ou étrangers, des laboratoires publics ou privés. 


\title{
Nosé-Andersen dynamics of partially rigid molecules: Coupling all degrees of freedom to heat and pressure baths
}

\author{
G. R. Kneller* \\ Institut für Theoretische Physik A, Rheinisch-Westfälische Technische Hochschule Aachen, \\ Templergraben 55, D-52056 Aachen, Germany \\ T. Mülders ${ }^{\dagger}$ \\ Institut für Biochemie, Rheinisch-Westfälische Technische Hochschule Aachen, \\ Klinikum Pauwelsstraße 30, D-52057 Aachen, Germany
}

(Received 31 May 1996)

\begin{abstract}
We derive the equations of motion for partially rigid molecules in the isothermal-isobaric $N-P-T$ ensemble The extended system method introduced by Andersen [J. Chem. Phys. 72, 2384 (1980)] and Nosé [J. Chem. Phys. 81, 511 (1984)] is generalized to the case of discrete mechanical systems subject to geometrical constraints. In our approach all available degrees of freedom are coupled to both the heat and the pressure bath. In this way we take into account that partially rigid molecules can adapt their conformation to volume fluctuations without violating intramolecular constraints. Using projector techniques and Dirac's theory of constrained Hamiltonian dynamics, we derive the equations of motion in Cartesian coordinates and extend the generalized Euler equations for linked rigid bodies [G. R. Kneller and K. Hinsen, Phys. Rev. E 50, 1559 (1994)] to the case of $N-P-T$ dynamics. We prove that the trajectories generated by our equations of motion correspond to the desired $N-P-T$ ensemble. [S1063-651X(96)12711-2]

PACS number(s): 03.20.+i, 02.70.-c
\end{abstract}

\section{INTRODUCTION}

Andersen introduced the "extended system method" in order to perform molecular-dynamics (MD) simulations in the isobaric-isoenthalpic $N-P-H$ ensemble [1] $(N, P$, and $H$ denote constant particle number, constant pressure, and constant enthalpy, respectively). Here the pressure bath is described by an additional dynamical variable, and the dynamics of the whole system, i.e., the physical system plus the additional variable, is described in the framework of Hamiltonian mechanics. The introduction of the extended system method was an important step in the history of computer simulations since completely deterministic MD simulations could now be performed in other ensembles than the "natural" microcanonical $N-V-E$ ensemble, in which the volume $V$ and the total energy $E$ are constants. Nosé showed that the extended system method allows as well the generation of trajectories corresponding to the canonical $N-V-T$ ensemble ( $T$ is the temperature) and the isothermal-isobaric $N-P-T$ ensemble, which is appropriate for most experimental situations [2]. In [1] Andersen treated also the case of the $N-P-T$ ensemble, but the temperature was controlled by a stochastic method. The original extended system method was designed for simulations of simple liquids. Later Ryckaert, Ciccotti, and Ferrario presented extensions for simulations of molecular liquids [3-7]. The idea was to preserve the correctness of the simulated ensembles in the presence of geometrical constraints. The generalization of Anderson's method for systems subject to holonomic constraints is not straightforward

\footnotetext{
*Electronic address: g.kneller@kfa-juelich.de

†Electronic address: thomas@bionm1.biochem.rwth-aachen.de
}

since the latter are destroyed by the space scaling method that is used to adjust the pressure. Ryckaert, Ciccotti, and Ferrario circumvented this problem by coupling Andersen's pressure bath only to the center-of-mass positions of the molecules. Since velocity scaling does not affect holonomic constraints, it is easy to implement the Nosé thermostat in a simulation program for partially rigid molecules such that all degrees of freedom couple to the temperature bath. This is also desirable for Andersen's barostat since the local response of the system due to pressure steering is kept as small as possible in this way.

In principle, a general and elegant method to combine thermodynamic and geometrical constraints is offered by Gauss's principle of least constraint [8-12]. In contrast to Hamilton's variational principle of mechanics, Gauss's principle is a time-local minimum principle for the accelerations of the particles, which can be derived from d'Alembert's principle of virtual displacements. The proof is given by Gauss in his original article [8]. His idea was to formulate constrained mechanics as a least-squares problem. Evans et al. recognized the value of Gauss's principle to set up equations of motion for arbitrary position and velocitydependent constraints. The latter cannot be properly handled in the framework of Hamiltonian mechanics [13]. Gauss's principle allows, for instance, one to construct a thermostat by fixing the kinetic energy or to impose an average particle flow describing a nonequilibrium system [14]. One can as well impose constant pressure and constant temperature [15]. For an overview see also [16] and [17]. The combination of geometrical constraints with a Gauss thermostat has been used in polymer simulations by Edberg, Evans, and Morriss [18]. Here the thermostat was coupled only to the centers of mass of the molecules, but not to all atomic degrees of freedom, which is possible as well, but complicates the equations 
for the Lagrange multipliers associated with the constraints. As soon as explicit kinematical conditions can be formulated, as in nonequilibrium molecular dynamics, Gauss's principle offers a mathematically clear and simple way to write down the equations of motion. A priori it is, however, not obvious which constraints are to be imposed in order to describe a specific thermodynamic ensemble. The Gauss thermostat, e.g., fixes the kinetic energy and not the average kinetic energy, which is constant in the canonical ensemble. Correspondingly, the phase-space distribution is of the usual form $e^{-\beta \mathcal{V}(\mathbf{r})}$ in configurational space, but in velocity space it yields a $\delta$ function centered at the prescribed kinetic energy $[2,19]$. We are not aware that the ensemble corresponding to a Gauss thermostat and/or barostat in the presence of geometrical constraints has been identified. Berendsen et al. [20] suggested a third method for MD simulations at constant temperature and/or constant pressure that is known as the "weak-coupling scheme." They use controllers with proportional characteristics [17] to maintain temperature and pressure at prescribed values. Geometrical constraints are handled separately via the SHAKE method [21]. By construction, all degrees of freedom couple to the heat and pressure bath (weak coupling). However, even for systems without geometrical constraints the resulting trajectories cannot be shown to correspond to a well-defined thermodynamic ensemble [22]. The latter is indispensable if one wishes to obtain the correct fluctuations of thermodynamic variables. On the other hand, the method by Ryckaert, Ciccotti, and Ferrario is not suitable if only a few but large molecules are to be simulated at constant pressure. In the extreme case where the simulated system consists of only one long polymer chain it cannot be applied at all since pressure adjustment by center-of-mass scaling becomes meaningless.

In this paper we focus on the simulation of partially rigid molecules in the conventional thermodynamic $N-P-T$ ensemble. We show how the extended system method can be generalized to simulations of discrete mechanical systems with arbitrary holonomic constraints such that all available degrees of freedom are coupled to the heat and the pressure bath (see Fig. 1). In this way not only the distances between the molecules, but also their internal coordinates respond to volume fluctuations. The latter is reflected in a change of the radius of gyration. In Sec. II we set up the Nosé-Andersen Lagrangian corresponding to a geometrically constrained system and construct the corresponding Hamiltonian. Using the Dirac theory of constrained Hamiltonian dynamics [2325], we derive first the Hamiltonian equations of motion in Cartesian coordinates (Sec. III). Then we adapt the generalized Euler equations for linked rigid bodies [26] to the case of $N-P-T$ dynamics (Sec. IV). In Sec. $\mathrm{V}$ we give the proof that our equations of motion correspond to the desired $N-P-T$ ensemble. An important result is also that we obtain a microscopic expression for the pressure in a system of partially rigid molecules. This aspect is discussed in Sec. VI. A concluding discussion follows in Sec. VII and in the Appendix details on general coordinates in virtual and real space are given. Numerical considerations and an application are planned to be discussed in a future paper.

\section{CONSTRAINED EXTENDED SYSTEMS}

\section{A. Geometrical constraints and projectors}

We consider a dynamical system consisting of $N$ pointlike particles whose positions are determined by $3 N$ mass-

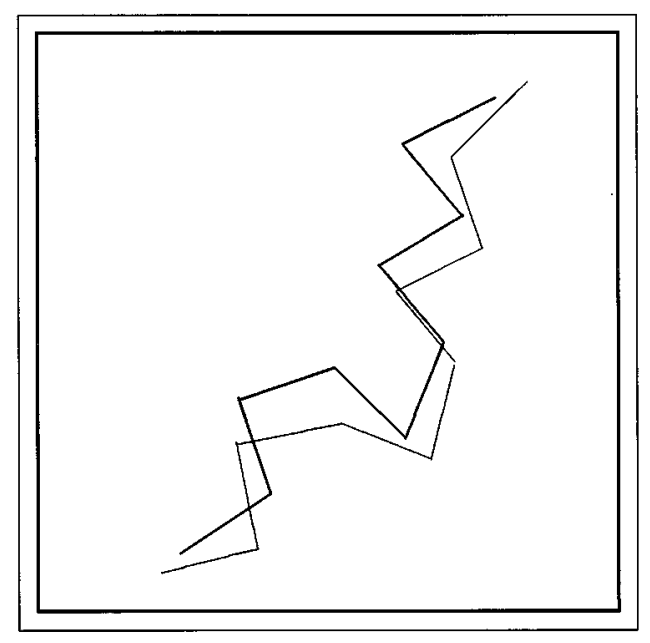

FIG. 1. Stretching of a molecule by enlargement of the simulation box. The bond lengths in the original molecule (thick lines) and in the stretched molecule (thin lines) are the same; only the angles have changed.

weighted Cartesian coordinates $\mathbf{r}=\left(r^{1}, \ldots, r^{3 N}\right)^{T}$. The components of $\mathbf{r}$ are the particle positions multiplied by the square roots of their masses. To keep the notation short mass-weighted coordinates and related quantities will not be labeled as such. The relations between mass-weighted and non-mass-weighted quantities are listed in Table I. If coupling to pressure and temperature baths is disregarded, the dynamics of our system is governed by the Lagrangian

$$
\mathcal{L}=\frac{1}{2} \dot{\mathbf{r}}^{T} \dot{\mathbf{r}}-\mathcal{V}(\mathbf{r})
$$

and $l$ holonomic constraints

$$
\sigma^{\alpha}(\mathbf{r}) \equiv 0, \quad \alpha=1, \ldots, l .
$$

The latter describe, for instance, chemical bonds, planar rings, or a reaction coordinate that is constrained to a certain value. We introduce now the $l \times 3 N$ matrix $\mathbf{A}$, whose elements are the partial derivatives of the constraints $\sigma^{\alpha}$ with respect to the particle positions,

$$
A_{j}^{\alpha}(\mathbf{r})=\frac{\partial \sigma^{\alpha}(\mathbf{r})}{\partial r^{j}}, \quad \alpha=1, \ldots, l, \quad j=1, \ldots, 3 N
$$

In the following we will make extensive use of the projectors on the null space of $\mathbf{A}$ and its orthogonal complement. These projectors will be denoted as $\mathcal{P}_{\|}$and $\mathcal{P}_{\perp}$, respectively. They can be concisely expressed in terms of the matrix $\mathbf{A}$ :

TABLE I. Mass weighting.

\begin{tabular}{ccc}
\hline \hline Mass weighted & Not mass weighted & Connection \\
\hline $\mathbf{r}$ & $\mathbf{r}^{*}$ & $\mathbf{r}^{*}=\mathbf{M}^{-1 / 2} \mathbf{r}$ \\
$\mathbf{p}$ & $\mathbf{p}^{*}$ & $\mathbf{p}^{*}=\mathbf{M}^{1 / 2} \mathbf{p}$ \\
$\mathbf{f}$ & $\mathbf{f}^{*}$ & $\mathbf{f}^{*}=\mathbf{M}^{1 / 2} \mathbf{f}$ \\
$\boldsymbol{\sigma}(\mathbf{r})=\mathbf{0}$ & $\boldsymbol{\sigma}^{*}\left(\mathbf{r}^{*}\right)=\mathbf{0}$ & $\boldsymbol{\sigma}(\mathbf{r})=\boldsymbol{\sigma}^{*}\left(\mathbf{r}^{*}\right)$ \\
$\mathbf{A}$ & $\mathbf{A}^{*}$ & $\mathbf{A}^{*}=\mathbf{A} \mathbf{M}^{1 / 2}$ \\
$\mathbf{z}$ & $\mathbf{z}^{*}$ & $\mathbf{z}^{*}=\mathbf{M}^{1 / 2} \mathbf{z}$ \\
$\mathbf{C}$ & $\mathbf{C}^{*}$ & $\mathbf{C}^{*}=\mathbf{M}^{-1 / 2} \mathbf{C}$ \\
\hline \hline
\end{tabular}




$$
\begin{gathered}
\mathcal{P}_{\|}=\mathbf{1}-\mathbf{A}^{+} \mathbf{A}, \\
\mathcal{P}_{\perp}=\mathbf{A}^{+} \mathbf{A} .
\end{gathered}
$$

$\mathbf{A}^{+}$is the Moore-Penrose inverse of $\mathbf{A}$ [27-29], which is also called the "generalized inverse" or "pseudoinverse." Assuming that $\mathbf{A}$ has full row rank, $\mathbf{A}^{+}$can be written in the explicit form [27]

$$
\mathbf{A}^{+}=\mathbf{A}^{T}\left(\mathbf{A} \mathbf{A}^{T}\right)^{-1} .
$$

Both $\mathcal{P}_{\|}$and $\mathcal{P}_{\perp}$ fulfill the relations $\mathcal{P}^{2}=\mathcal{P}$ and $\mathcal{P}^{T}=\mathcal{P}$. It is easy to see that $\mathbf{A} \mathcal{P}_{\|}=\mathbf{0}$, i.e., $\mathcal{P}_{\|}$is indeed the projector on the null space of $\mathbf{A}$. In the following the null space of $\mathbf{A}$ is called $V_{\|}$and its orthogonal complement is called $V_{\perp}$. Application of $\mathcal{P}_{\|}$and $\mathcal{P}_{\perp}$ to an arbitrary vector $\mathbf{x}$ yields its projections on $V_{\|}$and $V_{\perp}$, respectively. We denote the projection on $V_{\|}$by $\mathbf{x}_{\|}$and the one on $V_{\perp}$ by $\mathbf{x}_{\perp}$.

Time differentiation of the constraint equations (2) shows that the velocities are in the null space of $\mathbf{A}$,

$$
\mathbf{A} \mathbf{r}=\mathbf{0} \Leftrightarrow \mathcal{P}_{\|} \dot{\mathbf{r}}=\dot{\mathbf{r}}_{\|}=\dot{\mathbf{r}}
$$

It follows from the simple form of $\mathcal{L}$ that the canonical momenta are identical to the velocities

$$
\mathbf{p}=\frac{\partial \mathcal{L}}{\partial \dot{\mathbf{r}}}=\dot{\mathbf{r}}
$$

and thus fulfill the same constraints

$$
\mathbf{A p}=\mathbf{0} \Leftrightarrow \mathcal{P}_{\| \mathbf{p}}=\mathbf{p}_{\|}=\mathbf{p} .
$$

\section{B. Lagrangian of the extended system}

We make now the following ansatz for the NoséAndersen Lagrangian of the constrained extended system in mass-weighted coordinates:

$$
\begin{aligned}
\mathcal{L}_{v}= & \frac{Q^{2 / 3} s^{2}}{2} \dot{\boldsymbol{\rho}}^{T} \mathcal{P}_{\|}\left(Q^{1 / 3} \boldsymbol{\rho}\right) \dot{\boldsymbol{\rho}}-\mathcal{V}\left(Q^{1 / 3} \boldsymbol{\rho}\right)+\frac{s^{2} W_{Q}}{2} \dot{Q}^{2}+\frac{W_{s}}{2} \dot{s}^{2} \\
& -P_{\text {ext }} Q-g k_{B} T \ln s .
\end{aligned}
$$

The Lagrangian $\mathcal{L}_{v}$, together with kinematical conditions yet to be specified, describes the dynamics of a virtual system consisting of $N$ pointlike particles. $Q$ and $s$ are scaling variables. $Q$ has the dimension of volume and $s$ is dimensionless. $W_{Q}$ and $W_{s}$ are the corresponding "masses," which have to be regarded as adjustable parameters. The vector $\boldsymbol{\rho}$ contains the $3 N$ mass-weighted positions of the virtual particles that are normalized to a cube of volume $Q$. All overdots in (10) denote time derivatives with respect to the virtual time $\tau$. The function $\mathcal{V}$ denotes the potential energy, $P_{\text {ext }}$ is the external pressure, $g$ is an integer number still to be determined, and $k_{B} T$ is the Boltzmann constant multiplied by the absolute temperature. The relations between the variables describing the virtual system (briefly called "virtual variables") and the variables describing the real system to be simulated ("real variables") are given in Table II. They do not constitute a canonical transformation and therefore the real variables cannot be equivalently used in the derivation of Hamiltonian equations of motion. The Lagrangian $\mathcal{L}_{v}$ is
TABLE II. Connection between virtual and real quantities.

\begin{tabular}{lcc}
\hline \hline Virtual & Real & Connection \\
\hline$Q$ & $V$ & $Q=V$ \\
$\boldsymbol{\pi}_{Q}$ & $p_{V}$ & $\frac{\pi_{Q}}{s}=p_{V}$ \\
$s$ & $S$ & $s=S$ \\
$\boldsymbol{\pi}_{s}$ & $p_{S}$ & $\pi_{s}=p_{s}$ \\
$\boldsymbol{\rho}$ & $\mathbf{r}$ & $Q^{1 / 3} \boldsymbol{\rho}=\mathbf{r}$ \\
$\boldsymbol{\pi}$ & $\mathbf{p}$ & $\frac{\pi}{Q^{1 / 3} s}=\mathbf{p}$ \\
$\delta \tau$ & $d t$ & $\frac{d \tau}{s}=d t$ \\
\hline \hline
\end{tabular}

constructed in analogy to the Lagrangian of Nosé given in [2]. The main difference between $\mathcal{L}_{v}$ and the corresponding Lagrangian for $N$ unconstrained particles is the presence of the projector $\mathcal{P}_{\|}$. We include the velocity $\dot{Q}$ in the time scaling procedure to formulate the equations of motion in "Nosé-Hoover-like" form [19,7].

The constraints imposed on the virtual system read

$$
\sigma^{\alpha}\left(Q^{1 / 3} \boldsymbol{\rho}\right) \equiv 0, \quad \alpha=1, \ldots, l
$$

and the elements of the matrix $\mathbf{A}$ are now given by

$$
A_{i}^{\alpha}\left(Q^{1 / 3} \boldsymbol{\rho}\right)=\left.\frac{\partial \sigma^{\alpha}(\mathbf{r})}{\partial r^{i}}\right|_{\mathbf{r}=Q^{1 / 3} \boldsymbol{\rho}} .
$$

As in the case of real-space dynamics one can derive a constraint for the velocities by time differentiation of the kinematical conditions. One obtains from (11)

$$
\mathbf{A} \dot{\boldsymbol{\rho}}+\frac{\dot{Q}}{3 Q} \mathbf{A} \boldsymbol{\rho}=\mathbf{0}
$$

The solution of this equation for $\dot{\boldsymbol{\rho}}$ yields all virtual velocities that are kinematically possible,

$$
\dot{\boldsymbol{\rho}}=\underbrace{-\boldsymbol{\rho}_{\perp} \frac{\dot{Q}}{3 Q}}_{\dot{\boldsymbol{\rho}}_{\perp}}+\dot{\boldsymbol{\rho}}_{\|} .
$$

At this point $\dot{\boldsymbol{\rho}}_{\|}$is an arbitrary vector in $V_{\|}$. Equation (14) shows that $\dot{\boldsymbol{\rho}}$, in contrast to $\dot{\mathbf{r}}$, is not an eigenvector of $\mathcal{P}_{\|}$, but has also a component in $V_{\perp}$. If the projector $\mathcal{P}_{\|}$were inserted in the kinetic energy term of the real space Lagrangian $\mathcal{L}$ given in (1), the resulting trajectories would be exactly the same as the ones derived from $\mathcal{L}$ without the projector. In that light, singular Lagrangians in classical mechanics appear to be artificial [30]. However, $\mathcal{L}_{v}$ is truly singular. Here the presence of the projector does change the equations of motion. The virtual Lagrangian $\mathcal{L}_{v}$ is constructed in such a way that the virtual canonical momenta

$$
\boldsymbol{\pi}=\frac{\partial \mathcal{L}_{v}}{\partial \dot{\boldsymbol{\rho}}}
$$

fulfill the same constraints as the real momenta $\mathbf{p}$, 


$$
\mathbf{A} \boldsymbol{\pi}=\mathbf{0} \Leftrightarrow \mathcal{P}_{\|} \boldsymbol{\pi}=\boldsymbol{\pi}_{\|}=\boldsymbol{\pi} .
$$

The constraints for the coordinates and momenta describing the virtual system ensure that the real variables fulfill the conditions $\sigma^{\alpha}(\mathbf{r})=0$ and $\mathbf{A p}=\mathbf{0}$, respectively.

\section{Hamiltonian of the extended system}

The basic quantity that relates classical dynamics to statistical physics and thermodynamics is the Hamilton function. In order to generalize the extended system method to the case of semiflexible molecules we need a Hamiltonian description of a constrained system in Cartesian coordinates. First the Hamiltonian is needed to prove the correctness of the simulated ensemble, assuming the equivalence of time and ensemble averages. If mass-weighted coordinates are used, the Hamiltonian equations of motion have also a simpler form than the Lagrangian equations of motion. The theory of constrained Hamiltonian dynamics was established by Dirac [23,24] and Anderson and Bergmann [31]. De Leeuw, Perram, and Petersen treated this subject in a more recent paper [32].

To construct the Hamilton function associated with $\mathcal{L}_{v}$ we write

$$
\mathcal{H}_{v}=\dot{\boldsymbol{\rho}}^{T} \boldsymbol{\pi}+\dot{Q} \pi_{Q}+\dot{s} \pi_{s}-\mathcal{L}_{v} .
$$

$\pi, \pi_{Q}$, and $\pi_{s}$ are the virtual momenta, which are defined as $\partial \mathcal{L}_{v} / \partial \dot{\boldsymbol{\rho}}, \partial \mathcal{L}_{v} / \partial \dot{Q}$, and $\partial \mathcal{L}_{v} / \partial \dot{s}$, respectively. The velocities in $\mathcal{H}_{v}$ must now be eliminated in favor of the momenta. Since $\mathcal{L}_{v}$ contains the projector $\mathcal{P}_{\|}$as a metric tensor in the kinetic energy term, and consequently $\mathcal{P}_{\|} \boldsymbol{\pi}=\boldsymbol{\pi}$, the associated Hamiltonian $\mathcal{H}_{v}$ depends only on the component $\dot{\boldsymbol{\rho}}_{\|}$. The latter can be expressed as $\dot{\boldsymbol{\rho}}_{\|}=\pi / Q^{2 / 3} s^{2}$. The elimination of the remaining velocities $\dot{Q}$ and $\dot{s}$ is trivial and yields the following concise form for $\mathcal{H}_{v}$ :

$$
\begin{aligned}
\mathcal{H}_{v}= & \frac{1}{2 Q^{2 / 3} s^{2}} \boldsymbol{\pi}^{T} \boldsymbol{\pi}+\frac{\pi_{Q}^{2}}{2 s^{2} W_{Q}}+\frac{\pi_{s}^{2}}{2 W_{s}}+\mathcal{V}\left(Q^{1 / 3} \boldsymbol{\rho}\right)+P_{\mathrm{ext}} Q \\
& +g k_{B} T \ln s .
\end{aligned}
$$

On account of the momentum constraints $\mathcal{P}_{\|} \boldsymbol{\pi}=\boldsymbol{\pi}$, the projector $\mathcal{P}_{\|}$appearing in $\mathcal{L}_{v}$ can be omitted in $\mathcal{H}_{v}$. Compared to the Lagrangian equations of motion, where $\mathcal{P}_{\|}$must be kept, the Hamiltonian equations have a much simpler form. We note here that this is a result of the use of mass-weighted coordinates.

\section{EQUATIONS OF MOTION IN CARTESIAN COORDINATES}

\section{A. Hamilton's variational principle}

The Hamiltonian equations of motion for the virtual coordinates and momenta can be derived from the variational principle [11]

$$
\mathcal{S}=\int_{\tau_{0}}^{\tau_{1}} d \tau\left\{\dot{\boldsymbol{\rho}}^{T} \boldsymbol{\pi}+\dot{Q} \pi_{Q}+\dot{s} \pi_{s}-\mathcal{H}_{v}\right\}=\text { Extremum }
$$

This leads to the necessary condition

$$
\begin{aligned}
\delta \mathcal{S}= & -\int_{\tau_{0}}^{\tau_{1}} d \tau\left\{\boldsymbol{\delta} \boldsymbol{\rho}^{T}\left(\frac{\partial \mathcal{H}_{v}}{\partial \boldsymbol{\rho}}+\dot{\boldsymbol{\pi}}\right)+\boldsymbol{\delta} \boldsymbol{\pi}^{T}\left(\frac{\partial \mathcal{H}_{v}}{\partial \boldsymbol{\pi}}-\dot{\boldsymbol{\rho}}\right)\right. \\
& +\delta Q\left(\frac{\partial \mathcal{H}_{v}}{\partial Q}+\dot{\pi}_{Q}\right)+\delta \pi_{Q}\left(\frac{\partial \mathcal{H}_{v}}{\partial \pi_{Q}}-\dot{Q}\right)+\delta s\left(\frac{\partial \mathcal{H}_{v}}{\partial s}+\dot{\pi}_{s}\right) \\
& \left.+\delta \pi_{s}\left(\frac{\partial \mathcal{H}_{v}}{\partial \pi_{s}}-\dot{s}\right)\right\}=0 .
\end{aligned}
$$

The expressions in parentheses are to be evaluated at the true path that makes the action $\mathcal{S}$ stationary. It must be taken into account that the variations in (20) are restricted and interdependent due to the kinematic conditions for the virtual positions and momenta. Therefore the variations of the paths in configuration and momentum space are not arbitrary, and one cannot conclude that the terms in parentheses in (20) vanish identically. We discuss first the derivation of the equations of motion in Cartesian coordinates.

\section{B. Equations of motion for the virtual system}

The equations of motion for the virtual system can be derived by the Lagrange multiplier method. Following Dirac $[23,24]$, we observe that the Hamiltonian $\mathcal{H}_{v}$ is not uniquely determined. If we write the constraints for coordinates and momenta in the form $\Phi^{\beta} \equiv 0$, where

$$
\Phi^{\beta}=\left\{\begin{array}{l}
-\sigma^{\beta}\left(Q^{1 / 3} \boldsymbol{\rho}\right), \quad \beta=1, \ldots, l \\
\pi^{j} A_{j}^{\beta-l}\left(Q^{1 / 3} \boldsymbol{\rho}\right), \quad \beta=l+1, \ldots, 2 l,
\end{array}\right.
$$

we can introduce a new Hamiltonian

$$
\mathcal{H}_{v}^{*}=\mathcal{H}_{v}+c_{\beta} \Phi^{\beta}
$$

containing $2 l$ arbitrary functions $c_{\beta}(\tau)$. (Here and in the following we use the Einstein convention, assuming implicitly a summation over pairwise upper and lower indices. Upper indices refer to contravariant components and lower indices to covariant components. In a Euclidean coordinate system covariant and contravariant components are identical. In some places we distinguish formally between them to respect the Einstein convention.) Obviously $\mathcal{H}_{v}^{*}=\mathcal{H}_{v}$ on the surface defined by $\Phi^{\beta} \equiv 0$. For variations of the paths in phase space that are in accordance with the constraints it follows that $\delta \Phi^{\beta}=0$, and consequently $\delta \mathcal{H}_{v}^{*}=\delta \mathcal{H}_{v}$. However, the derivatives of $\mathcal{H}_{v}^{*}$ with respect to the momenta and coordinates yield additional terms $c_{\beta} \partial \Phi^{\beta} / \partial \boldsymbol{\pi}, c_{\beta} \partial \Phi^{\beta} / \partial \boldsymbol{\rho}$, etc. The Hamiltonian $\mathcal{H}_{v}^{*}$ allows us to consider an unconstrained variational problem, which is supplemented by the $2 l$ conditions $\Phi^{\beta} \equiv 0$. These allow us to fix the $c_{\beta}$ that appear now as Lagrange multipliers in complete analogy with Lagrange's equations of the first kind. To derive the Hamiltonian equations of motion we split the $c_{\beta}$ into a set of $l$ Lagrange multipliers $\left\{\mu_{\alpha}\right\}$ corresponding to the position constraints $\sigma^{\alpha} \equiv 0$ and a set of $l$ Lagrange multipliers $\left\{\gamma_{\alpha}\right\}$ corresponding to the momentum constraints $A_{i}^{\alpha} \pi^{i} \equiv 0$. Introducing the vectors $\boldsymbol{\sigma}=\left(\sigma^{1}, \ldots, \sigma^{l}\right)^{T}, \boldsymbol{\mu}=\left(\mu_{1}, \ldots, \mu_{l}\right)^{T}$, and $\boldsymbol{\gamma}=\left(\gamma_{1}, \ldots, \gamma_{l}\right)^{T}$, the Hamiltonian $\mathcal{H}_{v}^{*}$ may be expressed as

$$
\mathcal{H}_{v}^{*}=\mathcal{H}_{v}-\boldsymbol{\sigma}^{T}\left(Q^{1 / 3} \boldsymbol{\rho}\right) \boldsymbol{\mu}+\boldsymbol{\pi}^{T} \mathbf{A}^{T}\left(Q^{1 / 3} \boldsymbol{\rho}\right) \boldsymbol{\gamma}
$$


The equations of motion are now derived by inserting $\mathcal{H}_{v}^{*}$ in the stationarity condition (20) and considering an unconstrained variational problem. This means that all variations can be considered as independent.

To write the equations of motion in matrix notation we introduce the $3 N$-dimensional force vector $\mathbf{f}$ and the $l \times 3 N$ matrix $\mathbf{F}$ via

$$
\begin{gathered}
f_{i}=-\left.\frac{\partial \mathcal{V}}{\partial r^{i}}\right|_{\mathbf{r}=Q^{1 / 3} \boldsymbol{\rho}}, \quad i=1, \ldots, 3 N \\
F_{k}^{\alpha}=-\left.\pi^{i} \frac{\partial^{2} \sigma^{\alpha}}{\partial r^{k} \partial r^{i}}\right|_{\mathbf{r}=Q^{1 / 3} \boldsymbol{\rho}}, \quad \alpha=1, \ldots, l, \quad k=1, \ldots, 3 N .
\end{gathered}
$$

Using these definitions, the Hamiltonian equations of motion in virtual coordinates and virtual time are found to be

$$
\begin{aligned}
& \dot{\boldsymbol{\rho}}=\underbrace{\frac{\boldsymbol{\pi}}{Q^{2 / 3} s^{2}}}_{\dot{\boldsymbol{\rho}}}+\underbrace{\mathbf{A}^{T} \boldsymbol{\gamma}}_{\dot{\boldsymbol{\rho}}_{\perp}} \\
& \dot{\boldsymbol{\pi}}=Q^{1 / 3}(\mathbf{f}+\underbrace{\mathbf{A}^{T} \boldsymbol{\mu}}_{\mathbf{z}}+\mathbf{F}^{T} \boldsymbol{\gamma}) \\
& \dot{Q}=\frac{\pi_{Q}}{s^{2} W_{Q}}, \\
& \dot{\pi}_{Q}=-P_{\mathrm{ext}}+\frac{\boldsymbol{\pi}^{T} \boldsymbol{\pi}}{3 Q^{5 / 3} s^{2}}+\frac{\boldsymbol{\rho}^{T}}{3 Q^{2 / 3}}(\mathbf{f}+\underbrace{\mathbf{A}^{T} \boldsymbol{\mu}}_{\mathbf{z}}+\mathbf{F}^{T} \boldsymbol{\gamma}), \\
& \dot{s}=\frac{\pi_{s}}{W_{s}}, \\
& \dot{\pi}_{s}=\frac{\boldsymbol{\pi}^{T} \boldsymbol{\pi}}{Q^{2 / 3} s^{3}}+\frac{\pi_{Q}^{2}}{W_{Q} s^{3}}-\frac{g k_{B} T}{s} .
\end{aligned}
$$

The vector $\mathbf{z}=\mathbf{A}^{T} \boldsymbol{\mu}$ contains the yet undetermined massweighted constraint forces and the vector $\mathbf{A}^{T} \boldsymbol{\gamma}$ can be identified with the component $\dot{\boldsymbol{\rho}}_{\perp}$. This follows immediately from $\mathcal{P}_{\perp} \boldsymbol{\pi}=\mathbf{0}$ and $\mathcal{P}_{\perp} \mathbf{A}^{T}=\mathbf{A}^{T}$. In principle, both $\boldsymbol{\gamma}$ and $\boldsymbol{\mu}$ can be fixed by making use of the constraint equations $\sigma^{\alpha}\left(Q^{1 / 3} \boldsymbol{\rho}\right) \equiv 0$. For practical purposes, however, it is more convenient to determine only $\boldsymbol{\gamma}$ at this point, and to perform the computation of $\boldsymbol{\mu}$ in real-space coordinates. We know from Eq. (14) that $\dot{\boldsymbol{\rho}}_{\perp}=-(\dot{Q} / 3 Q) \boldsymbol{\rho}_{\perp}$. This allows us to write

$$
\dot{\boldsymbol{\rho}}_{\perp}=-\frac{\dot{Q}}{3 Q} \underbrace{\mathbf{A}^{+} \mathbf{A} \boldsymbol{\rho}}_{\mathcal{P}_{\perp}}=\mathbf{A}^{T} \boldsymbol{\gamma}
$$

where $\mathcal{P}_{\perp}=\mathbf{A}^{+} \mathbf{A}$ is the projector on the row space of $\mathbf{A}$. According to Eq. (6), the pseudoinverse of $\mathbf{A}$ has the explicit form $\mathbf{A}^{+}=\mathbf{A}^{T}\left(\mathbf{A A}^{T}\right)^{-1}$ if $\mathbf{A}$ is assumed to have full row rank. Multiplication of (32) by $\mathbf{A}$ from the left yields then a system of $l$ linear equations for the components of $\gamma$,

$$
\mathbf{A A}^{T} \boldsymbol{\gamma}=-\frac{\dot{Q}}{3 Q} \mathbf{A} \boldsymbol{\rho} .
$$

\section{Equations of motion of the real system}

To write down the equations of motion in real space we introduce the $l$-dimensional vector $\mathbf{g}$ such that

$$
\mathbf{A A}^{T} \mathbf{g}=-\mathbf{A} \mathbf{r}, \quad \boldsymbol{\gamma}=\left(\frac{1}{V^{1 / 3} S} \frac{\dot{V}}{3 V}\right) \mathbf{g}
$$

From now on the overdot denotes again a time derivative with respect to real time $t$. In addition, we define the symmetric $3 N \times 3 N$ matrix $\mathbf{H}$ with elements

$$
H_{i k}(\mathbf{r})=g_{\alpha} \frac{\partial^{2} \sigma^{\alpha}}{\partial r^{i} \partial r^{k}}, \quad i, k=1, \ldots, 3 N
$$

The $g_{\alpha}$ are the components of $\mathbf{g}$. The above definitions may now be inserted into the equations of motion in virtual coordinates, performing at the same time the substitutions listed in Table II. The result is

$$
\begin{gathered}
\dot{\mathbf{r}}=\frac{\dot{V}}{3 V} \mathbf{r}_{\|}+\mathbf{p}, \\
\dot{\mathbf{p}}=\mathbf{f}+\mathbf{z}-\frac{\dot{V}}{3 V}(\mathbf{1}+\mathbf{H}) \mathbf{p}-\zeta \mathbf{p}, \\
\dot{p}_{V}=-P_{\mathrm{ext}}+\frac{1}{3 V}\left\{\mathbf{p}^{T} \mathbf{p}+\mathbf{r}^{T}\left(\mathbf{f}+\mathbf{z}-\frac{p_{V}}{3 V}, \mathbf{H p}\right)\right\}-\zeta p_{V}, \\
\dot{\zeta}=\frac{1}{W_{V}}\left(\mathbf{p}^{T} \mathbf{p}+\frac{p_{V}^{2}}{W_{V}}-g k_{B} T\right) .
\end{gathered}
$$

Here we have introduced the "friction coefficient" $\zeta=\dot{S} / S$. The vector $\mathbf{g}$ can be used to compute $\mathbf{r}_{\|}$appearing in the equation of motion for $\mathbf{r}$. Writing $\mathbf{r}_{\|}=\mathcal{P}_{\|} \mathbf{r}$ $=\left[\mathbf{1}-\mathbf{A}^{T}\left(\mathbf{A} \mathbf{A}^{T}\right)^{-1} \mathbf{A}\right] \mathbf{r}$, it follows that

$$
\mathbf{r}_{\|}=\mathbf{r}+\mathbf{A}^{T} \mathbf{g}
$$

In addition to $\mathbf{r}_{\|}$, the constraint forces

$$
\mathbf{z}=\mathbf{A}^{T} \boldsymbol{\mu}
$$

need to be determined. A linear equation for $\boldsymbol{\mu}$ is obtained by time differentiation of the identity $\mathbf{A p}=\mathbf{0}$. This yields $\mathbf{A} \mathbf{p}=$ - $\mathbf{A} \mathbf{p}$. Here $\dot{\mathbf{p}}$ can be expressed by the right-hand side of Eq. (37). All terms proportional to $\mathbf{p}$ will drop out on account of $\mathbf{A p}=\mathbf{0}$, and we get

$$
\mathbf{A} \mathbf{A}^{T} \boldsymbol{\mu}=-\dot{\mathbf{A}} \mathbf{p}-\mathbf{A}\left(\mathbf{f}-\frac{\dot{V}}{3 V} \mathbf{H} \mathbf{p}\right) .
$$


It should be noted that the constraint forces depend explicitly on $\dot{V} / 3 V$, but not on $\zeta=\dot{S} / S$. This shows that a Nosé thermostat does not interfere with holonomic constraints.

From the equation for $\dot{p}_{V}$, which may be written in the concise form

$$
\dot{p}_{V}=P_{\text {inst }}(t)-P_{\text {ext }}-\zeta p_{V},
$$

the definition of the instantaneous pressure can be obtained:

$$
P_{\text {inst }}(t)=\frac{1}{3 V}\left\{\mathbf{p}^{T} \mathbf{p}+\mathbf{r}^{T}\left(\mathbf{f}+\mathbf{z}-\frac{\dot{V}}{3 V} \mathbf{H p}\right)\right\} .
$$

The expression for the mean pressure is derived in Sec. VI.

\section{EQUATIONS OF MOTION FOR LINKED RIGID BODIES}

\section{A. Linear velocity constraints}

In the following the generalized Euler equations of topologically linked rigid bodies [26,33] will be generalized to the case of $N-P-T$ dynamics. The $3 N$ real-space velocities of the $N$ pointlike constituents are related to a set of $f$ generalized velocities via

$$
\dot{\mathbf{r}}=\mathbf{C}(\mathbf{r}) \mathbf{u} \text {. }
$$

Consider a system of $L$ chain molecules, enumerated by $J=1, \ldots, L$, each containing $m_{J}$ rigid bodies. The vector $\mathbf{u}$ comprises then $L$ translational velocities and $M=\sum_{J=1}^{L} m_{J}$ angular velocities. To keep the formulas in the derivation of the equations of motion as simple as possible, we consider a single $C$ matrix for the whole system. In general, the $C$ matrix is not a Jacobian. This case is, of course, included. Since we are using mass-weighted coordinates we have $\mathbf{C}$ $=\mathbf{M}^{1 / 2} \mathbf{C}^{*}$, where $\mathbf{C}^{*}$ is the definition of the $C$ matrix in [26] and $\mathbf{M}$ is the diagonal mass matrix. The $C$ matrix depends on the Cartesian coordinates of the particles, which depend, in turn, on quaternion parameters describing the orientation of the rigid units. For each unit there are four normalized quaternion parameters. A linear relation of the form $\dot{\mathbf{q}}=\boldsymbol{\Lambda}(\mathbf{q}) \boldsymbol{\omega}$ connects the time derivatives of the quaternion parameters and the angular velocity of the respective unit.

\section{B. Hamilton's principle for linked rigid bodies}

Formally the geometrical constraints of the chain can be described in the alternative form $\sigma^{\alpha}(\mathbf{r})=0$. As shown above, the velocities in real space fulfill the condition $\mathbf{A} \dot{\mathbf{r}}=\mathbf{0}$, where the elements of $\mathbf{A}$ are the partial derivatives of the $\boldsymbol{\sigma}^{\alpha}$ with respect to the particle coordinates. Since $\dot{\mathbf{r}}=\mathbf{C u}$ and $\mathbf{u}$ is an arbitrary vector, it follows that $\mathbf{A C}=\mathbf{0}$. This shows that the columns of $\mathbf{C}$ span the null space of $\mathbf{A}$.

The equations of motion for linked rigid bodies in the $N-P-T$ ensemble are again derived from the stationarity condition (20). Now the variations $\boldsymbol{\delta} \boldsymbol{\rho}$ and $\boldsymbol{\delta} \boldsymbol{\pi}$ are constructed by using the basis vectors of $V_{\|}$. These are now explicitly given in form of the column vectors of the matrix $\mathbf{C}$.
To obtain an explicit expression for the variation $\boldsymbol{\delta} \boldsymbol{\rho}$ we proceed as follows. If the geometrical constraints in virtual coordinates are given in the form $\sigma^{\alpha}\left(Q^{1 / 3} \boldsymbol{\rho}\right)=0$, the variation of these equations yields [compare Eq. (14)]

$$
\mathbf{A} \boldsymbol{\delta} \boldsymbol{\rho}=-\frac{\delta Q}{3 Q} \mathbf{A} \boldsymbol{\rho} .
$$

The formal solution for $\boldsymbol{\delta} \boldsymbol{\rho}$ reads

$$
\boldsymbol{\delta} \boldsymbol{\rho}=-\frac{\delta Q}{3 Q} \boldsymbol{\rho}_{\perp}+\delta \rho_{\|}
$$

where $\delta \boldsymbol{\rho}_{\|}$is an arbitrary variation in $V_{\|}$. Since the columns of $\mathbf{C}$ form a basis of $V_{\|}$we can write

$$
\delta \boldsymbol{\rho}=\underbrace{-\boldsymbol{\rho}_{\perp} \frac{\delta Q}{3 Q}}_{\delta \boldsymbol{\rho}_{\perp}}+\underbrace{\mathbf{C}\left(Q^{1 / 3} \boldsymbol{\rho}\right) \delta \mathbf{y}}_{\delta \boldsymbol{\rho}_{\|}} .
$$

Here $\boldsymbol{\delta} \mathbf{y}$ is an arbitrary $f$-dimensional vector.

Since $\mathcal{P}_{\|} \boldsymbol{\pi}=\boldsymbol{\pi}$ the virtual momenta $\boldsymbol{\pi}$ can be written in the form

$$
\boldsymbol{\pi}=\mathbf{C}\left(Q^{1 / 3} \boldsymbol{\rho}\right) \mathbf{v} .
$$

The vector $\mathbf{v}$ contains $f$ virtual generalized velocities $(\mathbf{v}$ is not to be confused with $\mathbf{u}$ ). Since $\mathbf{C}$ depends on $\boldsymbol{\rho}$ and $Q$ the variations of $\boldsymbol{\pi}$ are given by

$$
\delta \pi=(\delta \mathbf{C}) \mathbf{v}+\underbrace{\mathbf{C} \delta \mathbf{v}}_{\delta \pi_{\|, f}} .
$$

$\boldsymbol{\delta} \mathbf{v}$ are arbitrary variations of the velocities $\mathbf{v}$ and $\boldsymbol{\delta} \boldsymbol{\pi}_{\|, f}$ is an arbitrary $3 N$-dimensional vector in $V_{\|}$. The vector $\boldsymbol{\delta} \boldsymbol{\pi}_{\|, f}$ is not the complete projection of the momentum variation $\boldsymbol{\delta} \pi$ onto $V_{\|}$, but only its free part, which is left undetermined by the momentum constraints. Since $\mathbf{C}$ depends on $Q$ and $\boldsymbol{\rho}$ its variation, $\boldsymbol{\delta} \mathbf{C}$ splits into a contribution proportional to $\delta Q$ and a second one that is proportional to $\boldsymbol{\delta} \boldsymbol{\rho}$. According to Eq. (49), the latter splits, in turn, into a contribution proportional to $\delta Q$ and a second one that is in $V_{\|}$. Written in components, $\delta \pi$ reads

$$
\begin{aligned}
\delta \pi^{i}= & Q^{1 / 3}(\underbrace{-\frac{\delta Q \rho_{\perp}^{k}}{3 Q}}_{\delta \rho_{\perp}^{k}}+\underbrace{C_{\beta}^{k} \delta h^{\beta}}_{\delta \rho_{\|}^{k}}) \frac{\partial C_{\alpha}^{i}}{\partial\left(Q^{1 / 3} \rho^{k}\right)} v^{\alpha} \\
& +\frac{\delta Q \rho^{k}}{3 Q^{2 / 3}} \frac{\partial C_{\alpha}^{i}}{\partial\left(Q^{1 / 3} \rho^{k}\right)} v^{\alpha}+\underbrace{C_{\alpha}^{i} \delta v^{\alpha}}_{\delta \pi_{\|, f}^{i}} .
\end{aligned}
$$

The expressions for $\boldsymbol{\delta} \boldsymbol{\rho}$ and $\boldsymbol{\delta} \boldsymbol{\pi}$, as given by Eqs. (49) and (52), respectively, are now to be used in the stationarity condition (20). More explicitly we get 


$$
\begin{aligned}
\delta \mathcal{S}= & -\int_{\tau_{0}}^{\tau_{1}} d \tau\left(\delta \pi_{\|, f}^{i}\left(\frac{\partial \mathcal{H}_{v}}{\partial \pi^{i}}-\dot{\rho}_{i}\right)\right. \\
& +\delta \rho_{\|}^{k}\left(\frac{\partial \mathcal{H}_{v}}{\partial \rho^{k}}+\dot{\pi}_{k}+Q^{1 / 3}\left[\frac{\partial \mathcal{H}_{v}}{\partial \pi^{i}}-\dot{\rho}_{i}\right] \frac{\partial C_{\alpha}^{i}}{\partial\left(Q^{1 / 3} \rho^{k}\right)} v^{\alpha}\right) \\
& +\delta \pi_{Q}\left(\frac{\partial \mathcal{H}_{v}}{\partial \pi_{Q}}-\dot{Q}\right)+\delta Q\left\{\frac{\partial \mathcal{H}_{v}}{\partial Q}+\dot{\pi}_{Q}+\frac{1}{3 Q^{2 / 3}}\right. \\
& \times\left[\left(\rho^{k}-\rho_{\perp}^{k}\right)\left(\frac{\partial \mathcal{H}_{v}}{\partial \pi^{i}}-\dot{\rho}_{i}\right)\right] \frac{\partial C_{\alpha}^{i}}{\partial\left(Q^{1 / 3} \rho^{k}\right)} v^{\alpha} \\
& \left.-\frac{\rho_{\perp}^{k}}{3 Q}\left[\frac{\partial \mathcal{H}_{v}}{\partial \rho^{k}}+\dot{\pi}_{k}\right]\right\}+\delta \pi_{s}\left(\frac{\partial \mathcal{H}_{v}}{\partial \pi_{s}}-\dot{s}\right) \\
& \left.+\delta s\left(\frac{\partial \mathcal{H}{ }_{v}}{\partial s}+\dot{\pi}_{s}\right)\right)=0
\end{aligned}
$$

The first point to be observed is that $\boldsymbol{\delta} \boldsymbol{\rho}_{\|}, \boldsymbol{\delta} \boldsymbol{\pi}_{\|, f}, \delta Q, \delta \pi_{Q}$, $\delta s$, and $\delta \pi_{s}$ are the independent variations. In addition, one must take into account that $\boldsymbol{\delta} \boldsymbol{\pi}_{\|, f}$ and $\boldsymbol{\delta} \boldsymbol{\rho}_{\|}$are both restricted to the subspace $V_{\|}$, which is spanned by the columns of $\mathbf{C}$. Writing the corresponding stationarity conditions as $\boldsymbol{\delta} \boldsymbol{\pi}_{\|, f}^{T}$ $\left(\mathcal{E}_{1}\right)=0$ and $\boldsymbol{\delta} \boldsymbol{\rho}_{\|}^{T}\left(\mathcal{E}_{2}\right)=0$, it follows that $\mathcal{E}_{1}$ and $\mathcal{E}_{2}$ must be vectors in $V_{\perp}$. We call them $-\dot{\boldsymbol{\rho}}_{\perp}$ and $Q^{1 / 3} \mathbf{z}$, respectively. The factor $Q^{1 / 3}$ in front of $\mathbf{z}$ has been introduced to give $\mathbf{z}$ the dimension of a force. Since $\delta Q, \delta \pi_{Q}, \delta s$, and $\delta \pi_{s}$ are unrestricted, the associated terms in parentheses must vanish identically.

\section{Equations of motion in virtual and real space}

To write the equations of motion for linked rigid bodies in matrix form we introduce the $3 N \times f$ matrix $\mathbf{G}$,

$$
G_{k \alpha}=\left.\left(\dot{\rho}_{\perp}\right)_{i} \frac{\partial C_{\alpha}^{i}}{\partial r^{k}}\right|_{\mathbf{r}=Q^{1 / 3} \boldsymbol{\rho}}, \quad k=1, \ldots, 3 N, \quad \alpha=1, \ldots, f .
$$

After straightforward algebra the equations of motion in virtual coordinates are found to take the form

$$
\begin{gathered}
\dot{\boldsymbol{\rho}}=\frac{\mathbf{C} \mathbf{v}}{Q^{2 / 3} s^{2}}+\dot{\boldsymbol{\rho}}_{\perp}, \quad \dot{\boldsymbol{\rho}}_{\perp}=-\boldsymbol{\rho}_{\perp} \frac{\dot{Q}}{3 Q}, \\
\dot{\boldsymbol{\pi}}=\mathbf{C} \dot{\mathbf{v}}+\dot{\mathbf{C}} \mathbf{v}=Q^{1 / 3}(\mathbf{f}+\mathbf{z})+Q^{1 / 3} \mathbf{G} \mathbf{v}, \\
\dot{Q}=\frac{\pi_{Q}}{W_{Q^{s}}{ }^{2}}, \\
\dot{\pi}_{Q}=-P_{\mathrm{ext}}+\frac{\mathbf{v}^{T} \mathbf{C}^{T} \mathbf{C v}}{3 Q^{5 / 3} s^{2}}+\frac{\boldsymbol{\rho}^{T}}{3 Q^{2 / 3}}(\mathbf{f}+\mathbf{z}+\mathbf{G v}), \\
\dot{s}=\frac{\pi_{s}}{W_{s}}, \\
\dot{\pi}_{s}=\frac{\mathbf{v}^{T} \mathbf{C}^{T} \mathbf{C v}}{Q^{2 / 3} s^{3}}+\frac{\pi_{Q}^{2}}{W_{Q} s^{3}}-\frac{g k_{B} T}{s} .
\end{gathered}
$$

To derive the equation of motion for $\pi_{Q}$ we used that $\boldsymbol{\rho}_{\perp}^{T} \mathbf{z}=\boldsymbol{\rho}^{T} \mathbf{z}$, which follows from $\mathbf{z} \in V_{\perp}$. The vector $\mathbf{f}$ contains again the forces and $\mathbf{z}$ comprises the constraint forces. An equation of motion for $\mathbf{v}$ is obtained by multiplying (56) from the left by $\mathbf{C}^{+}$. Assuming that all column vectors in $\mathbf{C}$ are linearly independent, the relations

$$
\mathbf{C}^{+}=\left(\mathbf{C}^{T} \mathbf{C}\right)^{-1} \mathbf{C}^{T}, \quad \mathbf{C}^{+} \mathbf{C}=\mathbf{1}
$$

hold. Since $\mathbf{C}^{T} \mathbf{z}=\mathbf{0}$ the constraint forces on the right-hand side of the equation for $\dot{\boldsymbol{\pi}}$ drop out and one obtains

$$
\dot{\mathbf{v}}=\mathbf{C}^{+}\left(Q^{1 / 3} \mathbf{f}-\dot{\mathbf{C}} \mathbf{v}+Q^{1 / 3} \mathbf{G v}\right) .
$$

If the dynamics of a constrained system is described in Cartesian coordinates the connection between all dynamical variables in virtual and real space is given by the simple scaling relations listed in Table II. This is no longer true if generalized coordinates and velocities are used. To establish the relation between the variables appearing in the virtual equations of motion and the angular velocities describing the real system we introduce another set of $f$ auxiliary velocities, comprised in the vector $\mathbf{w}=\left(w^{1}, \ldots, w^{f}\right)^{T}$,

$$
\mathbf{w}=V^{-1 / 3} S^{-1} \mathbf{v},
$$

The components of $\mathbf{w}$ have the same dimension as those of $\mathbf{u}$, but $\mathbf{w}$ cannot be identified with $\mathbf{u}$. It is now convenient to define the $3 N \times f$ matrix $\mathbf{K}$ through

$$
K_{j \alpha}=\left(r_{\perp}\right)_{i} \frac{\partial C_{\alpha}^{i}(\mathbf{r})}{\partial r^{j}}, \quad j=1, \ldots, 3 N, \quad \alpha=1, \ldots, f .
$$

To obtain $\mathbf{r}_{\perp}$ we write

$$
\mathbf{r}_{\perp}=\mathbf{r}-\mathbf{r}_{\|}=\mathbf{r}-\mathbf{C h},
$$

where $\mathbf{h}$ is to be determined from the requirement that $\mathbf{C}^{T} \mathbf{r}_{\perp}=0$. The resulting equation for $\mathbf{h}$ is

$$
\mathbf{C}^{T} \mathbf{C h}=\mathbf{C}^{T} \mathbf{r} \text {. }
$$

Using the definitions for $\mathbf{w}$ and $\mathbf{K}$ together with the relations in Table II, the equations of motion in real coordinates are found to be

$$
\begin{aligned}
& \mathbf{u}=\mathbf{w}+\frac{\dot{V}}{3 V} \mathbf{C}^{+} \mathbf{r} \\
& \dot{\mathbf{w}}=\mathbf{C}^{+}(\mathbf{f}-\dot{\mathbf{C}} \mathbf{w})-\frac{\dot{V}}{3 V}\left(\mathbf{1}+\mathbf{C}^{+} \mathbf{K}\right) \mathbf{w}-\zeta \mathbf{w} \\
& \dot{V}=\frac{p_{V}}{W_{V}}, \\
& \dot{p}_{V}=-P_{\text {ext }}+\frac{1}{3 V}\left\{\mathbf{w}^{T} \mathbf{C}^{T} \mathbf{C w}+\mathbf{r}^{T}\left(\mathbf{f}+\mathbf{z}-\frac{\dot{V}}{3 V} \mathbf{K w}\right)\right\}-\zeta p_{V}, \\
& \dot{\zeta}=\frac{1}{W_{s}}\left(\mathbf{w}^{T} \mathbf{C}^{T} \mathbf{C w}+\frac{p_{V}^{2}}{2 W_{V}}-g k_{B} T\right) .
\end{aligned}
$$


Here the overdots are derivatives with respect to real time $t$. We used again the definition $\zeta=\dot{S} / S$ for the friction coefficient. Some comments on the relation between $\mathbf{u}$ and $\mathbf{w}$ need to be given here. From the equation for $\dot{\boldsymbol{\rho}}$ one obtains first $\dot{\mathbf{r}}=\mathbf{C w}+(\dot{V} / 3 V) \mathbf{r}_{\|}$. Since the velocities can be expressed as $\dot{\mathbf{r}}=\mathbf{C u}$ it follows that $\mathbf{C u}=\mathbf{C w}+(\dot{V} / 3 V) \mathbf{r}_{\|}$. This equation is now multiplied by $\mathbf{C}^{+}$. Using that $\mathbf{C}^{+} \mathbf{C}=\mathbf{1}$, it follows that $\mathbf{u}=\mathbf{w}+(\dot{V} / 3 V) \mathbf{C}^{+} \mathbf{r}_{\|}$. Since $\mathbf{C}^{T} \mathbf{r}_{\perp}=0$, the vector $\mathbf{C}^{+} \mathbf{r}_{\|}$may be replaced by $\mathbf{C}^{+} \mathbf{r}$, which leads to Eq. (67).

The equations of motion are not yet complete since the constraint forces are still unknown. To find an expression for $\mathbf{z}$ we transform the equation for $\dot{\boldsymbol{\pi}}$ into real-space coordinates

$$
\mathbf{C} \dot{\mathbf{w}}+\dot{\mathbf{C}} \mathbf{w}=\mathbf{f}+\mathbf{z}-\frac{\dot{V}}{3 V}(\mathbf{C}+\mathbf{K}) \mathbf{w}-\zeta \mathbf{C w} .
$$

We know that the constraint forces are in the null space of $\mathbf{C}$ such that $\mathcal{P}_{\perp} \mathbf{z}=\left(\mathbf{1}-\mathbf{C} \mathbf{C}^{+}\right) \mathbf{z}=\mathbf{z}$. Multiplication of the above equation by $\mathcal{P}_{\perp}$ yields then an equation for $\mathbf{z}$. Here we can use that the projector $\mathcal{P}_{\perp}$ annihilates all terms proportional to C. The result is

$$
\mathbf{z}=\mathcal{P}_{\perp}\left(\dot{\mathbf{C}} \mathbf{w}+\frac{\dot{V}}{3 V} \mathbf{K} \mathbf{w}-\mathbf{f}\right)
$$

The vector on the right-hand side can be obtained in the same way as $\mathbf{r}_{\perp}$.

\section{Rigid rotors}

We will now show that in the case of an ensemble of rigid rotors the volume scaling affects only the centers of mass, as it should be. In principle, the degrees of freedom of the whole system could be described by a single $C$ matrix. For the following proof, however, it is more convenient to introduce separate $C$ matrices for each rotor and to decompose the coordinates into center-of-mass and relative coordinates. We introduce the vectors $\boldsymbol{\rho}_{J, \text { c.m. }}$, containing the three Cartesian components of the center of mass of rotor $J$ and the vectors $\boldsymbol{\rho}_{J}^{\prime}$, collecting for each rotor the relative coordinates of all atoms with respect to its center of mass. The virtual position of atom $k$ in rotor $J$ is then given by

$$
\boldsymbol{\rho}_{J, k}=\boldsymbol{\rho}_{J, \mathrm{c} . \mathrm{m} .}+\boldsymbol{\rho}_{J, k}^{\prime} .
$$

In terms of center-of-mass and relative coordinates the virtual Lagrangian reads

$$
\begin{aligned}
\mathcal{L}_{v}= & \sum_{J=1}^{L} \frac{Q^{2 / 3} s^{2}}{2} \dot{\boldsymbol{\rho}}_{J, \mathrm{c} . \mathrm{m} .}^{T} \dot{\boldsymbol{\rho}}_{J, \mathrm{c} . \mathrm{m} .} \\
& +\sum_{J=1}^{L} \frac{Q^{2 / 3} s^{2}}{2} \dot{\boldsymbol{\rho}}_{J}^{\prime T} \mathcal{P}_{J, \|}^{\prime}\left(Q^{1 / 3} \boldsymbol{\rho}_{J}^{\prime}\right) \dot{\boldsymbol{\rho}}_{J}^{\prime} \\
& -\mathcal{V}\left(\left\{Q^{1 / 3} \boldsymbol{\rho}_{J, \mathrm{c} . \mathrm{m} .}+Q^{1 / 3} \boldsymbol{\rho}_{J, k}^{\prime}\right\}\right)+\frac{s^{2} W_{Q}}{2} \dot{Q}^{2}+\frac{W_{s}}{2} \dot{s}^{2} \\
& -P_{\mathrm{ext}} Q-g k_{B} T \ln s .
\end{aligned}
$$

The important point is now that the constraints depend only on the relative coordinates

$$
\sigma_{J}^{\alpha}\left(Q^{1 / 3} \boldsymbol{\rho}_{J}^{\prime}\right)=0
$$

The whole procedure of deriving the equations of motion can now be repeated as described in the previous sections. Since the constraints involve only relative positions, the variations of the center-of-mass positions and momenta are to be considered as unrestricted. Analogously to the virtual center-ofmass coordinates $\boldsymbol{\rho}_{\text {c.m. }, J}$ and the virtual relative coordinates $\boldsymbol{\rho}_{J}^{\prime}$, we define $\mathbf{r}_{J, \text { c.m. }}$ and $\mathbf{r}_{J}^{\prime}$ to be the corresponding vectors in real space. The relative position of atom $k$ in rotor $J$ is denoted by $\mathbf{r}_{J, k}^{\prime}$. To indicate that the individual $C$ matrices depend only on relative coordinates, we label them as $\mathbf{C}_{J}^{\prime}$. They have the simple form [26]

$$
\mathbf{C}_{J}^{\prime}=\left(\begin{array}{c}
-\mathbf{R}_{J, 1}^{\prime} \\
\vdots \\
-\mathbf{R}_{J, m_{j}}^{\prime}
\end{array}\right)
$$

where the individual blocks are the skew symmetric matrices

$$
\mathbf{R}_{J, i}^{\prime}=\left(\begin{array}{rrr}
0 & -z_{J, i}^{\prime} & y_{J, i}^{\prime} \\
z_{J, i}^{\prime} & 0 & -x_{J, i}^{\prime} \\
-y_{J, i}^{\prime} & x_{J, i}^{\prime} & 0
\end{array}\right) .
$$

The above form for the $\mathbf{C}_{J}^{\prime}$ reflects the relation $\dot{\mathbf{r}}_{J, k}^{\prime}$ $=\omega_{J} \wedge \mathbf{r}_{J, k}^{\prime}$ and holds in Cartesian coordinates as well as in mass-weighted Cartesian coordinates. It is now easy to verify that

$$
\begin{gathered}
\mathbf{C}_{J}^{\prime T} \mathbf{r}_{J}^{\prime}=\mathbf{0}, \\
\mathbf{K}_{J}^{\prime}=-\mathbf{C}_{J}^{\prime},
\end{gathered}
$$

where for each rotor $J$ the matrix $\mathbf{K}_{J}^{\prime}$ is defined through Eq. (64), replacing $\mathbf{C}$ by $\mathbf{C}_{J}^{\prime}$. Using these relations one finds that

$$
\boldsymbol{\omega}_{J}=\mathbf{w}_{J}, \quad J=1, \ldots, M,
$$

where $\mathbf{w}_{J}$ is the molecular analog to the auxiliary velocity $\mathbf{w}$ defined in Eq. (63). It is then straightforward to show that the equations of motion in real coordinates read

$$
\begin{gathered}
\dot{\mathbf{r}}_{J, \mathrm{c} . \mathrm{m} .}=\mathbf{p}_{J, \mathrm{c} . \mathrm{m} .}+\frac{\dot{V}}{3 V} \mathbf{r}_{J, \mathrm{c} . \mathrm{m} .} \\
\dot{\mathbf{p}}_{J, \mathrm{c} . \mathrm{m} .}=\mathbf{f}_{J, \mathrm{c} . \mathrm{m} .}-\frac{\dot{V}}{3 V} \mathbf{p}_{J, \mathrm{c} . \mathrm{m} .}-\zeta \mathbf{p}_{J, \mathrm{c} . \mathrm{m} .}, \\
\dot{\boldsymbol{\omega}}_{J}=\mathbf{C}_{J}^{\prime+}\left(\mathbf{f}_{J}-\dot{\mathbf{C}}_{J}^{\prime} \boldsymbol{\omega}_{J}\right)-\zeta \boldsymbol{\omega}_{J}, \\
\dot{p}_{V}=-P_{\mathrm{ext}}+\frac{p_{V}}{3 V} \sum_{J=1}^{M}\left(\mathbf{p}_{J, \mathrm{c} . \mathrm{m} .}^{T} \mathbf{p}_{J, \mathrm{c} . \mathrm{m} .}+\mathbf{r}_{J, \mathrm{c} . \mathrm{m} .}^{T} \mathbf{f}_{J, \mathrm{c} . \mathrm{m} .}\right)-\zeta p_{V},
\end{gathered}
$$


$\dot{\zeta}=\frac{1}{W_{s}}\left(\sum_{J=1}^{M}\left(\mathbf{p}_{J, \text { c.m. }}^{T} \mathbf{p}_{J, \text { c.m. }}+\omega_{J}^{T} \mathbf{C}_{J}^{\prime T} \mathbf{C}_{J}^{\prime} \boldsymbol{\omega}_{J}\right)+\frac{p_{V}^{2}}{2 W_{V}}-g k_{B} T\right)$

The momenta $\mathbf{p}_{J, \text { c.m. }}$ are associated with the center-of-mass positions $\mathbf{r}_{J, \text { c.m. }}$. The vector $\mathbf{f}_{J, \text { c.m. }}$ denotes the total force on rotor $J, \mathbf{f}_{J, \mathrm{c} . \mathrm{m} .}=\Sigma_{k} \mathbf{f}_{J, k}$, where $\mathbf{f}_{J, k}$ are the forces on the individual atoms. Since $\mathcal{P}_{\perp, J}^{\prime} \mathbf{K}_{J}^{\prime}=-\mathcal{P}_{\perp, J}^{\prime} \mathbf{C}_{J}^{\prime}=\mathbf{0}$, the constraint forces are given by [compare Eq. (73)]

$$
\mathbf{z}_{J}=\mathcal{P}_{\perp, J}^{\prime}\left(\dot{\mathbf{C}}_{J}^{\prime} \boldsymbol{\omega}_{J}-\mathbf{f}_{J}\right)
$$

To derive the above equations of motion it was used that for rigid bodies the relation

$$
\mathbf{r}_{J}^{T} \mathbf{r}_{J}=\text { const }
$$

holds. This leads to $\mathbf{r}_{J}^{T}\left(\mathbf{f}_{J}+\mathbf{z}_{J}\right)=-\boldsymbol{\omega}_{J}^{T} \mathbf{C}_{J}^{\prime T} \mathbf{C}_{J}^{\prime} \boldsymbol{\omega}_{J}$ and simplifies the equation for $\dot{p}_{V}$ considerably. The equations of motion for the angular velocities do not couple to the volume dynamics and the instantaneous pressure is determined by the center-of-mass variables only. In the particular case of an ensemble of completely rigid molecules we retrieve the center of mass scaling procedure of Ryckaert, Ciccotti, and Ferrario [3-7]. If the Nosé thermostat is switched off, the rotational dynamics is described by the Euler equations of motion [26].

\section{CORRECTNESS OF THE ENSEMBLE}

It will now be shown that the equations of motion derived above correspond to the desired $N-P-T$ ensemble. As usual, the equivalence of time and ensemble averages is assumed.

\section{A. $\mathcal{H}_{v}$ is a constant of motion}

As a first step we show that the Hamiltonian $\mathcal{H}_{v}$ is a constant of motion. For this purpose it is convenient to collect all dynamical variables of the virtual system $\{\boldsymbol{\rho}, Q, s\}$ in the set $\left\{q^{\alpha}\right\}(\alpha=1, \ldots, 3 N+2)$ and all corresponding momenta in the set $\left\{p_{\alpha}\right\}(\alpha=1, \ldots, 3 N+2)$. The equations of motion (26) -(31) can then be written in the compact form

$$
\begin{gathered}
\dot{q}^{\alpha}=\frac{\partial \mathcal{H}_{v}^{*}}{\partial p_{\alpha}}, \\
\dot{p}_{\alpha}=-\frac{\partial \mathcal{H}_{v}^{*}}{\partial q^{\alpha}},
\end{gathered}
$$

where $\mathcal{H}_{v}^{*}=\mathcal{H}_{v}+c_{\beta} \Phi^{\beta}$. Using the above form of the equations of motion it follows immediately that $\dot{\mathcal{H}}_{v}^{*}=\dot{q}^{\alpha} \partial \mathcal{H}_{v}^{*}$ / $\partial q^{\alpha}+\dot{p}_{\alpha} \partial \mathcal{H}_{v}^{*} / \partial p_{\alpha}=0$, i.e., $\mathcal{H}_{v}^{*}$ is a constant of motion. Since the constraints vanish identically on the constraint surface $\Phi^{\beta} \equiv 0$, it follows that $\dot{\Phi}^{\beta}$ and all higher time derivatives vanish too. Consequently, $\mathcal{H}_{v}=\mathcal{H}_{v}^{*}-c_{\beta} \Phi^{\beta}$ is a constant of motion $\mathcal{H}_{v}=E$. Since $d / d t=s d / d \tau$, this holds also in real time, although the equations of motion in real space and time are no longer Hamiltonian equations of motion.

\section{B. Equivalence of the microcanonical ensemble} of the extended system and the NPT ensemble

To calculate ensemble averages we need to introduce generalized coordinates and momenta. We start with the definition (10) for the Lagrangian $\mathcal{L}_{v}$ of the constrained extended system. It has been shown above [see Eq. (14)] that the virtual velocities have the general form

$$
\dot{\boldsymbol{\rho}}=\underbrace{-\boldsymbol{\rho}_{1} \frac{\dot{Q}}{3 Q}}_{\dot{\boldsymbol{\rho}}_{\perp}}+\dot{\boldsymbol{\rho}}_{\|} .
$$

Note that the component $\dot{\boldsymbol{\rho}}_{\perp}$ does not contribute to the kinetic energy term in $\mathcal{L}_{v}$. We assume now having found a set of $f$ generalized coordinates $\eta^{\beta}$, collected in the vector $\boldsymbol{\eta}$ $=\left(\eta^{1}, \ldots, \eta^{f}\right)^{T}$ such that $\boldsymbol{\rho}$ can be parametrized as $\boldsymbol{\rho}(Q, \boldsymbol{\eta})$. Explanations on generalized coordinates in virtual and real space are given in the Appendix. The virtual velocities are then given by

where

$$
\dot{\boldsymbol{\rho}}=\underbrace{-\boldsymbol{\rho}_{\perp} \frac{\dot{Q}}{3 Q}}_{\dot{\boldsymbol{\rho}}_{\perp}}+\underbrace{\boldsymbol{\Gamma} \dot{\boldsymbol{\eta}}}_{\dot{\boldsymbol{m}}}
$$

$$
\Gamma_{\beta}^{i}=\frac{\partial \rho^{i}(Q, \boldsymbol{\eta})}{\partial \eta^{\beta}}, \quad-\frac{1}{3 Q} \rho_{\perp}^{i}=\frac{\partial \rho^{i}(Q, \boldsymbol{\eta})}{\partial Q} .
$$

The $\rho_{\perp}^{i}$ are the components of $\boldsymbol{\rho}_{\perp}=\mathcal{P}_{\perp} \boldsymbol{\rho} . \quad \boldsymbol{\Gamma}$ is a $3 N \times f$ matrix whose column vectors form a basis of the null space of $\mathbf{A}$ such that $\mathbf{A} \boldsymbol{\Gamma}=\mathbf{0}$. In terms of $\boldsymbol{\Gamma}$ the projector $\mathcal{P}_{\|}$reads

$$
\mathcal{P}_{\|}=\boldsymbol{\Gamma} \boldsymbol{\Gamma}^{+}=\boldsymbol{\Gamma}\left(\boldsymbol{\Gamma}^{T} \boldsymbol{\Gamma}\right)^{-1} \boldsymbol{\Gamma}^{T} .
$$

The above explicit form for $\mathcal{P}_{\|}$and expression (92) for $\dot{\boldsymbol{\rho}}$ may now be used to write $\mathcal{L}_{v}$ as

$$
\begin{aligned}
\mathcal{L}_{v}^{g}= & \frac{Q^{2 / 3} s^{2}}{2} \dot{\boldsymbol{\eta}}^{T} \boldsymbol{\Gamma}^{T} \boldsymbol{\Gamma} \dot{\boldsymbol{\eta}}+\frac{W_{Q s^{2} \dot{Q}^{2}}}{2}+\frac{W_{s} \dot{s}^{2}}{2} \\
& -\mathcal{V}-g k_{B} T \ln s-P_{\text {ext }} Q,
\end{aligned}
$$

where $\mathcal{V}=\mathcal{V}\left(Q^{1 / 3} \boldsymbol{\rho}[Q, \boldsymbol{\eta}]\right)$. The superscript $g$ stands for " generalized coordinates." As usual, the conjugate momenta to $\boldsymbol{\eta}$ are obtained via

$$
\boldsymbol{\pi}_{\eta}=\frac{\partial \mathcal{L}_{v}}{\partial \dot{\boldsymbol{\eta}}}=Q^{2 / 3} s^{2} \boldsymbol{\Gamma}^{T} \boldsymbol{\Gamma} \dot{\boldsymbol{\eta}},
$$

and we find that the corresponding Hamilton function is given by

$$
\begin{aligned}
\mathcal{H}_{v}^{g}= & \frac{1}{2 Q^{2 / 3} s^{2}} \boldsymbol{\pi}_{\eta}^{T}\left(\boldsymbol{\Gamma}^{T} \boldsymbol{\Gamma}\right)^{-1} \boldsymbol{\pi}_{\eta}+\frac{\pi_{Q}^{2}}{2 W_{Q} s^{2}}+\frac{\pi_{s}^{2}}{2 W_{s}}+\mathcal{V} \\
& +g k_{B} T \ln s+P_{\text {ext }} Q .
\end{aligned}
$$

To confirm that $\mathcal{H}_{v}^{g}$ is $\mathcal{H}_{v}$ in generalized coordinates, we need the relation between the Cartesian momenta $\boldsymbol{\pi}$ and the generalized momenta $\boldsymbol{\pi}_{\eta}$. This relation is easily established by using that $\boldsymbol{\pi}=\partial \mathcal{L}_{v} / \partial \dot{\boldsymbol{\rho}}=Q^{2 / 3} s^{2} \dot{\boldsymbol{\rho}}_{\|}=Q^{2 / 3} s^{2} \boldsymbol{\Gamma} \dot{\boldsymbol{\eta}}$. It follows then from Eq. (96) that

$$
\pi_{\eta}=\Gamma^{T} \pi
$$


If this is inserted in expression (97) one retrieves $\mathcal{H}_{v}$, observing that the projector $\mathcal{P}_{\|}$can be finally omitted on account of the momentum constraints $\mathcal{P}_{\|} \boldsymbol{\pi}=\boldsymbol{\pi}$.

Consider now the ensemble average of an arbitrary function $A(\mathbf{r}, \mathbf{p}, V)$ in the micro-canonical ensemble of the virtual system

$$
\langle A\rangle=\frac{\int \cdots \int d Q d \pi_{Q} d^{f} \boldsymbol{\pi}_{\eta} d^{f} \boldsymbol{\eta} d s d \pi_{s} \delta\left(\mathcal{H}_{v}^{g}-E\right) A(\mathbf{r}, \mathbf{p}, V)}{\int \cdots \int d Q d \pi_{Q} d^{f} \boldsymbol{\pi}_{\eta} d^{f} \boldsymbol{\eta} d s d \pi_{s} \delta\left(\mathcal{H}_{v}^{g}-E\right)} .
$$

$\mathcal{H}_{v}^{g}$ is the Hamilton function in generalized coordinates and $E$ is the constant energy of the virtual extended system. Using the relations in Table II, $\mathbf{r}, \mathbf{p}$, and $V$ can be written as

$$
\begin{gathered}
\mathbf{r}=Q^{1 / 3} \boldsymbol{\rho}(Q, \boldsymbol{\eta}), \\
\mathbf{p}=\frac{1}{Q^{1 / 3} s} \boldsymbol{\pi}=\frac{1}{Q^{1 / 3} S}\left(\boldsymbol{\Gamma}^{T}\right)^{+} \boldsymbol{\pi}_{\eta}, \\
V=Q .
\end{gathered}
$$

To express $\boldsymbol{\pi}$ in terms of $\boldsymbol{\pi}_{\eta}$ we inverted the equation $\boldsymbol{\pi}_{\eta}$ $=\boldsymbol{\Gamma}^{T} \boldsymbol{\pi}$, observing that $\mathcal{P}_{\|} \boldsymbol{\pi}=\boldsymbol{\pi}$.

As in the original Nosé-Andersen proof of the correctness of the ensemble $[1,2]$, we perform now a change to integration variables describing the real system. In the case of constrained motion Cartesian coordinates and momenta are to replaced by corresponding generalized (but not canonical) coordinates and momenta. The real-space positions are described by a set of $f=3 N-l$ generalized coordinates $x^{\alpha}$, collected in the vector $\mathbf{x}=\left(x^{1}, \ldots, x^{f}\right)^{T}$, such that $\mathbf{r}=\mathbf{r}(\mathbf{x})$. We recall that $f$ is the number of degrees of freedom, $l$ the number of constraints, and $N$ the number of particles. A comparison to (100) shows that there must be a relation $\mathbf{x}=\mathbf{x}(Q, \boldsymbol{\eta})$ such that

$$
\mathbf{r}(\mathbf{x}[Q, \boldsymbol{\eta}])=Q^{1 / 3} \boldsymbol{\rho}(Q, \boldsymbol{\eta}) .
$$

Obviously $\mathbf{r}$ is redundantly parametrized by the $f+1$ variables $\boldsymbol{\eta}$ and $Q$. This point is discussed in the Appendix. Analogously to the matrix $\boldsymbol{\Gamma}$, which is formed by the partial derivatives $\partial \rho^{i} / \partial \eta^{\alpha}$, we introduce the Jacobian $\mathbf{C}$ with elements

$$
C_{\alpha}^{i}=\frac{\partial r^{i}}{\partial x^{\alpha}}
$$

It follows from $\mathbf{A} \mathbf{r}=\mathbf{0}$ that the columns of $\mathbf{C}$ form a basis of the null space of $\mathbf{A}$. Write $A_{i}^{\alpha} \dot{r}^{i}=A_{i}^{\alpha}\left(\partial r^{i} / \partial x^{\beta}\right) \dot{x}^{\beta}$ $=A_{i}^{\alpha} C_{\beta}^{i} \dot{x}^{\beta}=0$. The velocities $\dot{x}^{\beta}$ are unrestricted and therefore $\mathbf{A C}=\mathbf{0}$. Since the columns of $\mathbf{C}$ and $\boldsymbol{\Gamma}$ span the same subspace $V_{\|}$, they must be related by a nonsingular linear transformation. The relation between $\boldsymbol{\Gamma}$ and $\mathbf{C}$ is found by writing

$$
\boldsymbol{\rho}(Q, \boldsymbol{\eta})=Q^{-1 / 3} \mathbf{r}(\mathbf{x}[Q, \boldsymbol{\eta}])
$$

and differentiating $\boldsymbol{\rho}$ with respect to $\boldsymbol{\eta}$. This yields [see Eq. (A3)]

$$
\boldsymbol{\Gamma}=Q^{-1 / 3} \mathbf{C B}, \quad B_{\beta}^{\alpha}(Q, \boldsymbol{\eta})=\frac{\partial x^{\alpha}(Q, \boldsymbol{\eta})}{\partial \eta^{\beta}} .
$$

The new set of variables is now chosen to be

$$
\begin{gathered}
V=Q, \\
p_{V}=\pi_{Q} / s, \\
S=s, \\
p_{S}=\pi_{s}, \\
\mathbf{x}=\mathbf{x}(Q, \boldsymbol{\eta}), \\
\mathbf{p}_{x}=\frac{1}{s}\left[\mathbf{B}^{T}(Q, \boldsymbol{\eta})\right]^{-1} \boldsymbol{\pi}_{\eta} .
\end{gathered}
$$

As stated in Eq. (106), B is the Jacobian for the change from $\boldsymbol{\eta}$ to $\mathbf{x}$. The volume elements transform as

$$
d Q d \pi_{Q} d^{f} \boldsymbol{\pi}_{\eta} d^{f} \boldsymbol{\eta} d s d \pi_{s}=S^{f+1} d V d p_{V} d^{f} \mathbf{p}_{x} d^{f} \mathbf{x} d s d p_{S}
$$

and the Hamiltonian $\mathcal{H}_{v}$ takes the form

$$
\begin{aligned}
\mathcal{H}_{v}^{g^{\prime}}= & \underbrace{\frac{1}{2} \mathbf{p}_{x}^{T}\left(\mathbf{C}^{T} \mathbf{C}\right)^{-1} \mathbf{p}_{x}+\mathcal{V}(\mathbf{r}[\mathbf{x}])}_{\mathcal{H}^{g}}+\frac{p_{V}^{2}}{2 W_{V}}+P_{\text {ext }} V+\frac{p_{S}^{2}}{2 W_{S}} \\
& +g k_{B} T \ln S .
\end{aligned}
$$

Here we have renamed $W_{V} \equiv W_{Q}, W_{S} \equiv W_{s} . \mathcal{H}^{g}$ is the Hamiltonian of the constrained real system in generalized coordinates. This is seen by expressing the Lagrangian $\mathcal{L}$ $=\frac{1}{2} \mathbf{r}^{T} \dot{\mathbf{r}}-\mathcal{V}$ in terms of generalized coordinates $\mathcal{L}^{g}=\frac{1}{2} \dot{\mathbf{x}}^{T} \mathbf{C}^{T} \mathbf{C} \dot{\mathbf{x}}-\mathcal{V}$, which yields the canonical momenta $\mathbf{p}_{x}=\mathbf{C}^{T} \mathbf{C} \dot{\mathbf{x}}$ and the Hamiltonian $\mathcal{H}^{g}=\frac{1}{2} \mathbf{p}_{x}^{T}\left(\mathbf{C}^{T} \mathbf{C}\right)^{-1} \mathbf{p}_{x}+\mathcal{V}$. It is worth noting that the Cartesian form corresponding to $\mathcal{H}^{g}$ is found by expressing $\mathbf{p}_{x}$ in terms of the Cartesian momenta $\mathbf{p}$. According to Eq. (98), one has $\boldsymbol{\pi}_{\eta}=\boldsymbol{\Gamma}^{T} \boldsymbol{\pi}$. Writing $\boldsymbol{\Gamma}, \boldsymbol{\pi}$, and $\boldsymbol{\pi}_{\eta}$ in terms of $\mathbf{C}, \mathbf{p}$, and $\mathbf{p}_{x}$, respectively, shows that

$$
\mathbf{p}_{x}=\mathbf{C}^{T} \mathbf{p} .
$$

Recognizing that $\mathcal{P}_{\|}=\mathbf{C}\left(\mathbf{C}^{T} \mathbf{C}\right)^{-1} \mathbf{C}^{T}$, we obtain

$$
\mathcal{H}^{g} \rightarrow \mathcal{H}=\frac{1}{2} \mathbf{p}^{T} \mathcal{P}_{\|} \mathbf{p}+\mathcal{V}=\frac{1}{2} \mathbf{p}^{T} \mathbf{p}+\mathcal{V} .
$$

Since $\dot{\mathbf{r}}=\mathbf{p}$ for purely geometrical constraints, this is exactly the Hamiltonian that results by a Legendre transformation of $\mathcal{L}=\frac{1}{2} \dot{\mathbf{r}}^{T} \dot{\mathbf{r}}-\mathcal{V}$.

We follow now Nosé and consider the denominator of formula (99) for the average of $A$. This denominator is the partition function $Z$. In terms of the variables $\left\{V, \ldots, \mathbf{p}_{x}\right\}$ we obtain

$$
Z=\int \cdots \int d V d p_{V} d^{f} \mathbf{p}_{x} d^{f} \mathbf{x} d s d p_{S} S^{f+2} \delta\left(\mathcal{H}_{v}^{g^{\prime}}-E\right) .
$$

The $\delta$ function can be written in the form $\delta\left(\mathcal{H}_{v}^{g^{\prime}}-E\right)$ $=\delta(h(S))$. Since $h(S)$ has one zero,

$$
S_{0}=\exp \left[-\left(\frac{1}{g k_{B} T}\right)\left(\mathcal{H}^{g}+\frac{p_{V}^{2}}{2 W_{V}}+P_{\text {ext }} V+\frac{p_{S}^{2}}{2 W_{S}}-E\right)\right],
$$


the integration over $S$ can be performed by using the identity $\delta(h(S))=1 /\left|h^{\prime}\left(S_{0}\right)\right| \delta\left(S-S_{0}\right)$. The result is

$$
\begin{aligned}
Z= & \int \cdots \int d V d p_{V} d^{f} \mathbf{p}_{x} d^{f} \mathbf{x} d p_{S} \exp \left[-\left(\frac{f+2}{g k_{B} T}\right) \mathcal{H}^{g}\right] \\
& \times \exp \left[-\left(\frac{f+2}{g k_{B} T}\right)\left(\frac{p_{V}^{2}}{2 W_{V}}+P_{\text {ext }} V\right)\right] \\
& \times \exp \left[-\left(\frac{f+2}{g k_{B} T}\right) \frac{p_{S}^{2}}{2 W_{S}}\right] \exp \left[\left(\frac{f+2}{g k_{B} T}\right) E\right] .
\end{aligned}
$$

The Gaussian integrals over $p_{S}$ and $p_{V}$ yield constants. If we set $g=f+2, Z$ is proportional to the partition function of the desired $N-P-T$ ensemble,

$$
Z \propto \iiint d V d^{f} \mathbf{p}_{x} d^{f} \mathbf{x} \exp \left[-\frac{\mathcal{H}^{g}+P_{\mathrm{ext}} V}{k_{B} T}\right] .
$$

This shows that

$$
\langle A\rangle=\langle A\rangle_{N P T},
$$

which is the desired result. Assuming the equivalence of time and ensemble averages, $\langle A\rangle$ corresponds to the time average $\bar{A}^{\tau}$ in virtual time. As shown by Nosé, sampling of $A$ in real time corresponds to the weighted average $\langle A / s\rangle /\langle 1 / s\rangle$. Therefore [see Eq. (117)] $g$ must be chosen as $g=f+1$ for real-time sampling [2] such that

$$
\langle A\rangle_{N P T}=\bar{A}^{t} .
$$

\section{PRESSURE}

The results of Sec. V B enable us to derive a microscopic expression for the average pressure, or simply the pressure.
Time averaging the equation of motion (39) for $p_{v}$ shows that $\overline{P_{\text {inst }}}=P_{\text {ext }}$ in equilibrium. Here $P_{\text {inst }}$ is the instantaneous pressure introduced in Eq. (45). Assuming the equivalence of time and ensemble averages we set $P_{\text {ext }}=\overline{P_{\text {inst }}}=\left\langle P_{\text {inst }}\right\rangle$. Since $\dot{V}=p_{V} / W_{V}, P_{\text {inst }}$ can be written in the form

$$
P_{\text {inst }}\left(p_{V}, V, \mathbf{p}, \mathbf{r}\right)=\frac{1}{3 V}\left\{\mathbf{p}^{T} \mathbf{p}+\mathbf{r}^{T}\left(\mathbf{f}+\mathbf{z}-\frac{p_{V}}{W_{V} 3 V} \mathbf{H p}\right)\right\} .
$$

The constraint forces $\mathbf{z}$ depend also on all four variables $\mathbf{p}, \mathbf{r}$, $p_{V}$, and $V$. Writing $\mathbf{z}=\mathbf{A}^{T} \boldsymbol{\mu}$ and using Eq. (43) for $\boldsymbol{\mu}$, we may formally write

$$
\mathbf{z}=-\mathbf{A}^{+}\left(\dot{\mathbf{A}} \mathbf{p}+\mathbf{A f}-\frac{p_{V}}{3 W_{V} V} \mathbf{H p}\right) .
$$

The corresponding expression for the case of linked rigid body dynamics is given in Eq. (73). In both cases the constraint forces depend explicitly and implicitly on $\dot{V} / 3 V=p_{V} / 3 W_{V} V$. The implicit dependence results from the time derivatives of $\mathbf{A}$ and $\mathbf{C}$, respectively. In the following we consider the case of Cartesian coordinates. In components we have $\dot{A}_{k}^{\alpha}=\dot{r}^{j} \partial A_{k}^{\alpha} / \partial r^{j}$, where $\dot{r}^{j}=p^{j}$ $+\left(p_{V} / 3 W_{V} V\right) r_{\|}^{j}$. This shows that the constraint forces contain two terms that depend both linearly on the momentum $p_{V}$. From expression (118) for the microcanonical partition function of the extended virtual system it follows now that the average $\left\langle P_{\text {inst }}\right\rangle$ can be expressed in the form

$$
\left\langle P_{\text {inst }}\right\rangle=\frac{\iiint \int d p_{V} d V d^{f} \mathbf{p}_{x} d^{f} \mathbf{x} w\left(p_{V}, V, \mathbf{p}_{x}, \mathbf{x}\right) P_{\text {inst }}\left(p_{V}, V, \mathbf{p}_{x}, \mathbf{x}\right)}{\iiint \int d p_{V} d V d^{f} \mathbf{p}_{x} d^{f} \mathbf{x} w\left(p_{V}, V, \mathbf{p}_{x}, \mathbf{x}\right)} .
$$

The weighting function $w$ is given by

$$
w\left(p_{V}, V, \mathbf{p}_{x}, \mathbf{x}\right)=\exp \left[-\frac{1}{k_{B} T}\left(\mathcal{H}^{g}\left(\mathbf{p}_{x}, \mathbf{x}\right)+\frac{p_{V}^{2}}{2 W_{V}}+P_{\text {ext }} V\right)\right] .
$$

All terms in $P_{\text {inst }}$ that are odd in $p_{V}$ will drop out since $w$ is even in $p_{V}$ and the integration range is symmetric with respect to zero. It remains now to give an expression for $\left\langle\mathbf{p}^{T} \mathbf{p}\right\rangle$. Writing

$$
\mathbf{p}^{T} \mathbf{p}=\mathbf{p}_{x}^{T}\left(\mathbf{C}^{T} \mathbf{C}\right)^{-1} \mathbf{p}_{x}=\mathbf{p}_{x}^{T} \frac{\partial \mathcal{H}^{g}}{\partial \mathbf{p}_{x}},
$$

one finds by integration over the momenta $\mathbf{p}_{x}$

$$
\left\langle\frac{\mathbf{p}^{T} \mathbf{p}}{3 V}\right\rangle=\left\langle\frac{f k_{B} T}{3 V}\right\rangle .
$$

The final expression for the pressure is thus

$$
P_{\mathrm{ext}}=\left\langle P_{\mathrm{inst}}\right\rangle=\left\langle\frac{f k_{B} T+\mathbf{r}^{T}\left(\mathbf{f}+\mathbf{z}_{g}\right)}{3 V}\right\rangle_{N P T} .
$$

The vector $\mathbf{z}_{g}$ contains the part of the constraint forces that does not depend on the volume dynamics, i.e., the purely geometrical constraint forces one would compute for given external forces $\mathbf{f}$ and velocities $\dot{\mathbf{r}} \equiv \mathbf{p}$. It is important to note that contributions from the constraint forces are to be considered in the pressure calculation [34].

\section{CONCLUSION}

We have presented a derivation of the equations of motion for discrete mechanical systems in the $N-P-T$ ensemble in the presence of holonomic constraints. Our approach represents a generalization of the extended system method in- 
troduced by Andersen and Nosé, which was designed for simple liquids. The main aspect is the generalization of Andersen's space scaling method for constant pressure simulations in the presence of holonomic constraints. Using Dirac's theory of constrained Hamiltonian dynamics, the equations of motion were derived for simulations in Cartesian coordinates. In addition, the generalized Euler equations of linked rigid bodies were extended to the case of $N-P-T$ dynamics. We have shown that our equations of motion correspond to the desired $N-P-T$ ensemble of a geometrically constrained system. For the special case of an ensemble of completely rigid molecules we retrieve the well-known center-of-mass scaling procedure [3-7]. However, for only partially rigid molecules the internal molecular degrees of freedom are coupled to the pressure bath. In this way local perturbations due to pressure controlling can be expected to dissipate more quickly. This effect is already known for temperature controlling with a Nosé thermostat.

It is important to note that the computation of constraint forces that maintain the imposed holonomic constraints cannot be decoupled from pressure steering if the system is to be simulated in the correct ensemble. This means that simulation methods that are commonly used to maintain geometrical constraints in Cartesian coordinates cannot be put "on top" of the Nosé-Andersen equations for unconstrained systems. The pressure is obtained from the usual viral expression, including the constraint forces. Here it is sufficient to use only the common geometrical constraint forces that are not identical to those actually used in the simulation of the dynamics.

Compared to unconstrained systems, the numerical integration of the equations of motion in Cartesian coordinates necessitates the following additional steps: (a) the computation of the projection $\mathbf{r}_{\|}$, (b) the computation of the second derivatives of the $\sigma^{\alpha}$ with respect to the particle positions, and (c) the computation of the constraint forces. To determine $\mathbf{r}_{\|}$one must solve a system of $l$ linear equations. The constraint forces may be determined by a SHAKE-like procedure, which ensures also that the geometrical constraints are exactly fulfilled. For bond constraints the second derivatives of the $\sigma^{\alpha}$ are simply constants, whereas they can be quite complicated expressions for more general constraints, such as angles. Programs for symbolic calculation are quite helpful in such a case.

The simulation of linked rigid bodies in the $N-P-T$ ensemble requires the solution of three systems of linear equations, each of which corresponds to a formal application of $\mathbf{C}^{+}$to a different right-hand side. The pseudoinverse $\mathbf{C}^{+}$is not needed explicitly. One of these matrix equations has the same form as the equation for the angular accelerations in the case of $N-V-E$ dynamics. In addition, the derivatives of the $C$ matrices with respect to the particle positions are to be provided. In contrast to the second derivatives of the $\sigma^{\alpha}$, they have always a simple form.

\section{APPENDIX}

Here we discuss the relation between generalized coordinates in real and in virtual space. We consider first the situation in real space where the Cartesian positions are restricted by $l$ constraints of the form $\sigma^{\alpha}(\mathbf{r})=0(\alpha=1, \ldots, l)$.
The remaining $f=3 N-l$ degrees of freedom may be described by the $f$ variables $\left\{x^{1}, \ldots, x^{f}\right\}$, with $x^{\beta}=x^{\beta}(\mathbf{r})$. Assuming, as usual, that the Jacobian $\left(\left[\partial x^{\beta} / \partial r^{i}\right] ;\left[\partial \sigma^{\alpha} / \partial r^{i}\right]\right)$ does not vanish, we can write $\mathbf{r}=\mathbf{r}(\mathbf{x} ; \boldsymbol{\sigma})$, abbreviating $\mathbf{x}=\left(x^{1}, \ldots, x^{f}\right)^{T}$ and $\boldsymbol{\sigma}=\left(\sigma^{1}, \ldots, \sigma^{l}\right)^{T}$. The $3 N$ components $r^{i}$ are the inverse functions of the $3 N$ functions $\left\{x^{\beta}, \sigma^{\alpha}\right\}$. Since $\boldsymbol{\sigma}=\mathbf{0}$, the vector $\mathbf{r}$ is a function of the $x^{\beta}$ alone and we write briefly $\mathbf{r}=\mathbf{r}(\mathbf{x})$. The differential of $\mathbf{r}$ reads

$$
\mathbf{d r}=\mathbf{C d x}, \quad C_{\beta}^{i}=\frac{\partial r^{i}}{\partial x^{\beta}} .
$$

The $f$ columns of $\mathbf{C}$ are the $f$ covariant basis vectors $\mathbf{c}_{\beta}=\partial \mathbf{r} / \partial x^{\beta}$. They are orthogonal to the $l$ contravariant basis vector $\mathbf{a}^{\alpha}=\partial \sigma^{\alpha} / \partial \mathbf{r}$. One way to see this is to use that the $\sigma^{\alpha}$ and the $x^{\beta}$ are independent dynamical variables and therefore $\partial \sigma^{\alpha} / \partial x^{\beta}=\left(\partial \sigma^{\alpha} / \partial r^{i}\right)\left(\partial r^{i} / \partial x^{\beta}\right)=0$. In matrix form we have $\mathbf{A C}=\mathbf{0}$, where the $\mathbf{a}^{\alpha}$ are the rows of $\mathbf{A}$. The columns of $\mathbf{C}$ span the subspace $V_{\|}$and the rows of $\mathbf{A}$ span the orthogonal complement $V_{\perp}$. We assume that both $\mathbf{A}$ and $\mathbf{C}$ have full rank.

The constraints in virtual variables $\operatorname{read} \sigma^{\alpha}\left(Q^{1 / 3} \boldsymbol{\rho}\right)=0$, which follows from $\mathbf{r}=Q^{1 / 3} \boldsymbol{\rho}$. Now $Q$ is supposed to be unrestricted: $a$ priori the volume can take any positive value. Therefore the equations $\sigma^{\alpha}\left(Q^{1 / 3} \boldsymbol{\rho}\right)=0$ are to be considered as $l$ constraints for $\boldsymbol{\rho}$, which depend parametrically on $Q$. We have $3 N$ Cartesian coordinates $\rho^{i}$ and $l$ constraints depending on $Q$, thus $f=3 N-l$ remaining degrees of freedom. A trivial parametrization of $\rho$ would be $\boldsymbol{\rho}(Q, \mathbf{x})=Q^{-1 / 3} \mathbf{r}(\mathbf{x})$. This corresponds to writing $x^{\beta}=x^{\beta}(\mathbf{r})=x^{\beta}\left(Q^{1 / 3} \boldsymbol{\rho}\right)$. In Sec. V we assumed the virtual position vector $\boldsymbol{\rho}$ to have the general form $\boldsymbol{\rho}=\boldsymbol{\rho}(Q, \boldsymbol{\eta})$. The differential $\mathbf{d} \boldsymbol{\rho}$ is then given by

$$
\mathbf{d} \boldsymbol{\rho}=\underbrace{-\boldsymbol{\rho}_{\perp} \frac{d Q}{3 Q}}_{d \boldsymbol{\rho}_{\perp}}+\underbrace{\boldsymbol{\Gamma} \mathbf{d} \boldsymbol{\eta}}_{d \boldsymbol{\rho}_{\|}}
$$

where $\Gamma_{\alpha}^{i}=\partial \rho^{i} / \partial \eta^{\alpha}$ and $\stackrel{d \boldsymbol{\rho}_{\perp}}{-}(1 / 3 Q) \rho_{\perp}^{i}=\partial \rho^{i} / \partial Q$. Differentiating now $\boldsymbol{\rho}=Q^{-1 / 3} \mathbf{r}(\mathbf{x})$ with respect to $Q$ yields $\partial \boldsymbol{\rho} / \partial Q$ $=-1 /(3 Q) \boldsymbol{\rho}$. But, in general, $\boldsymbol{\rho} \neq \boldsymbol{\rho}_{\perp}$ and relation (A2) would not be fulfilled. Therefore the set of variables $\{Q, \mathbf{x}\}$ is not suitable to describe the constrained dynamics of $\boldsymbol{\rho}$. To parametrize $\boldsymbol{\rho}$ we assume the $\eta^{\beta}$ to have the general form $\eta^{\beta}=\eta^{\beta}(Q, \boldsymbol{\rho})$. Together with the $\sigma^{\alpha}$ the $\eta^{\beta}$ form a complete set of $3 N$ functions of $\boldsymbol{\rho}$ that depends parametrically on $Q$ and may be inverted to give $\boldsymbol{\rho}=\boldsymbol{\rho}(Q, \boldsymbol{\eta})$. To find the relation between the variables $x^{\alpha}$ and $\eta^{\alpha}$ we write $\boldsymbol{\rho}$ $=Q^{-1 / 3} \mathbf{r}(\mathbf{x}[Q, \boldsymbol{\eta}])$. The differential form reads then

$$
\begin{aligned}
\mathbf{d} \boldsymbol{\rho}= & \left.\{-\frac{1}{3 Q} \boldsymbol{\rho}+Q^{-1 / 3} \underbrace{\frac{\partial \mathbf{r}}{\partial \mathbf{x}}}_{\mathbf{C}})\left(\frac{\partial \mathbf{x}}{\partial Q}\right)\right\} d Q \\
& +Q^{-1 / 3} \underbrace{\left.\frac{\partial \mathbf{r}}{\partial \mathbf{x}}\right)}_{\mathbf{C}} \underbrace{\left(\frac{\partial \mathbf{x}}{\partial \boldsymbol{\eta}}\right)}_{\mathbf{B}} \mathbf{d} \boldsymbol{\eta} .
\end{aligned}
$$

A comparison with expression (A2) shows that (a) $\boldsymbol{\Gamma}=Q^{-1 / 3} \mathbf{C B}$ and (b) the vector in curly brackets must be equal to $-1 /(3 Q) \boldsymbol{\rho}_{\perp}$. This leads to the condition $Q^{-1 / 3} \mathbf{C}(\partial \mathbf{x} / \partial Q)=(1 / 3 Q) \boldsymbol{\rho}_{\|}=(1 / 3 Q) \mathbf{C C}^{+} \boldsymbol{\rho}$. Here we used that 
the projector $\mathcal{P}$ on $V_{\|}$can be written as $\mathcal{P}=\mathbf{C} \mathbf{C}^{+}$. Since the columns of $\mathbf{C}$ are assumed to be linearly independent, we have $\mathbf{C}^{+}=\left(\mathbf{C}^{T} \mathbf{C}\right)^{-1} \mathbf{C}^{T}$. Performing the variable transformation $y=1 / 3 \ln Q$ we obtain

$$
\frac{\partial \mathbf{x}}{\partial y}=\left(\mathbf{C}^{T} \mathbf{C}\right)^{-1} \mathbf{C}^{T} \mathbf{r}=\mathbf{F}(\mathbf{x}(y))
$$

This is a system of $f$ ordinary differential equations for the $x^{\beta}$ as functions of $y$. Its solution contains $f$ " constants,", which can still be arbitrary functions of the $f$ variables $\eta^{\beta}$. For simple geometrical constraints (A4) can be solved analytically and shows that variables $\boldsymbol{\eta}$ leading to $\partial \boldsymbol{\rho} / \partial Q$ $=-1 /(3 Q) \boldsymbol{\rho}_{\perp}$ and $\partial \boldsymbol{\rho} / \partial \eta^{\beta} \in V_{\|}$can be explicitly constructed.

Having found a suitable parametrization $\boldsymbol{\rho}(Q, \boldsymbol{\eta})$, one can obviously write $\mathbf{r}=Q^{1 / 3} \boldsymbol{\rho}(Q, \boldsymbol{\eta})=\mathbf{r}(Q, \boldsymbol{\eta})$, where $\mathbf{r}$ depends on $\boldsymbol{\rho}$ and $Q$. This parametrization must be redundant since we know that $\mathbf{r}$ can be parametrized by $f$ variables $\left\{x^{1}, \ldots, x^{f}\right\}$. That this is indeed the case is readily seen. With $\mathbf{r}=Q^{1 / 3} \boldsymbol{\rho}$ we have from (A2)

$$
\mathbf{d r}=\frac{d Q}{3 Q^{2 / 3}} \boldsymbol{\rho}+Q^{1 / 3} \mathbf{d} \boldsymbol{\rho}=\frac{d Q}{3 Q} \mathbf{r}_{\|}+Q^{1 / 3} \boldsymbol{\Gamma} \mathbf{d} \boldsymbol{\eta}
$$

This is a linear combination of $f+1$ vectors in $V_{\|}$, which are $\mathbf{r}_{\|}$and the $f$ columns of $\boldsymbol{\Gamma}$. In an $f$-dimensional space only $f$ vectors can be linearly independent. This proves that the parametrization $\mathbf{r}=Q^{1 / 3} \boldsymbol{\rho}(Q, \boldsymbol{\eta})$ is redundant. Nevertheless, $\mathbf{r}$ is correctly parametrized since $\mathbf{d r}$ is in $V_{\|}$, as required by Eq. (A1).
[1] H. C. Andersen, J. Chem. Phys. 72, 2384 (1980).

[2] S. Nosé, J. Chem. Phys. 81, 511 (1984).

[3] J. P. Ryckaert and G. Ciccotti, J. Chem. Phys. 78, 7368 (1983).

[4] M. Ferrario and J. P. Ryckaert, Mol. Phys. 54, 587 (1985).

[5] J. P. Ryckaert and G. Ciccotti, Mol. Phys. 58, 1125 (1986).

[6] G. Ciccotti and J. P. Ryckaert, Comput. Phys. Rep. 4, 345 (1986).

[7] M. Ferrario, in Computer Simulation in Chemical Physics, Vol. 397 of NATO Advanced Study Institute, Series C: Mathematical and Physical Sciences, edited by M. P. Allen and D. J. Tildesley (Kluwer Academic, Dordrecht, 1993), pp. 153171.

[8] C. F. Gauss, J. Reine Angew. Math. 4, 232 (1829).

[9] E. Mach, The Science of Mechanics: A Critical and Historical Account of Its Development, 6th ed. (Open Court, Lasalle, IL, 1960).

[10] L. A. Pars, A Treatise on Analytical Dynamics (Heinemann, London, 1965).

[11] C. Lanczos, The Variational Principles of Mechanics, 4th ed. (University of Toronto Press, Toronto, 1974).

[12] E. T. Whittaker, A Treatise on the Analytical Dynamics of Particles and Rigid Bodies, 4th ed. (Cambridge University Press, London, 1970).

[13] H. Rund, The Hamilton-Jacobi Theory in the Calculus of Variations (Krieger, Huntington, NY, 1973).

[14] D. J. Evans, W. G. Hoover, B. H. Failor, B. Moran, and A. J. C. Ladd, Phys. Rev. A 28, 1016 (1983).

[15] D. J. Evans and G. P. Morris, Chem. Phys. 77, 63 (1983).

[16] D. J. Evans and G. P. Morris, Statistical Mechanics of Nonequilibrium Systems (Academic, New York, 1990).

[17] W. G. Hoover, in Molecular Dynamics, edited by H. Araki, J.
Ehlers, K. Hepp, R. Kippenhahn, H. A. Weidenmüller, and J. Zittartz, Lecture Notes in Physics Vol. 258 (Springer, Berlin, 1986).

[18] R. Edberg, D. Evans, and G. P. Morris, J. Chem. Phys. 84, 6933 (1986).

[19] W. G. Hoover, Phys. Rev. A 31, (1985).

[20] H. J. C. Berendsen, J. P. M. Postma, W. F. van Gunsteren, A. DiNola, and J. R. Haak, J. Chem. Phys. 81, 3684 (1984).

[21] J.-P. Ryckaert, G. Ciccotti, and H. J. C. Berendsen, J. Comput. Phys. 23, 327 (1977).

[22] M. P. Allen and D. J. Tildesley, Computer Simulation of Liquids (Oxford University Press, Oxford, 1987).

[23] P. A. M. Dirac, Canad. J. Math. 2, 129 (1950).

[24] P. A. M. Dirac, Proc. R. Soc. London Ser. A 246, 326 (1958).

[25] P. A. M. Dirac, Lectures on Quantum Mechanics (Yeshiva University, New York, 1964).

[26] G. R. Kneller and K. Hinsen, Phys. Rev. E 50, 1559 (1994).

[27] A. Ben-Israel and T. N. E. Greville, Generalized Inverses: Theory and Applications (Wiley, New York, 1974).

[28] E. H. Moore, Bull. Am. Math. Soc. 26, 394 (1920).

[29] R. Penrose, Proc. Cambridge Philos. Soc. 51, 406 (1955).

[30] K. Sundermeyer, in Constrained Dynamics, edited by $\mathrm{H}$. Araki, J. Ehlers, K. Hepp, and R. Kippenhahn, Lecture Notes in Physics Vol. 169 (Springer, Berlin, 1980).

[31] J. L. Anderson and P. G. Bergmann, Phys. Rev. 81, 1018 (1951).

[32] S. W. de Leeuw, J. W. Perram, and H. G. Petersen, J. Stat. Phys. 61, 1203 (1990).

[33] K. Hinsen and G. R. Kneller, Phys. Rev. E 52, 6868 (1995).

[34] W. Smith, Inf. Q. Comput. Simulat. Condens. Phases 39, 14 (1993). 